\title{
A new Geologic Time Scale, with special reference to Precambrian and Neogene
}

\author{
1. Geological Museum, University of Oslo, N-0318 Oslo, Norway.Email: felix.gradstein@nhm.uio.no \\ 2. Department of Earth \& Atmospheric Sciences, Purdue University, West Lafayette, Indiana 47907-1397, USA. \\ 3. Department of Earth Sciences, Cambridge University, Cambridge CB2 3EQ, England. \\ 4. Geological Survey of Canada, 601 Booth Str., Ottawa, Ontario K1A OE8, Canada. \\ 5. Faculty of Earth Sciences, Utrecht University, 3508 TA Utrecht, The Netherlands.
}

\begin{abstract}
A Geologic Time Scale (GTS2004) is presented that integrates currently available stratigraphic and geochronologic information. Key features of the new scale are outlined, how it was constructed, and how it can be further improved. The accompanying International Stratigraphic Chart, issued under auspices of the International Commission on Stratigraphy (ICS), shows the current chronostratigraphic scale and ages with estimates of uncertainty for all stage boundaries. Special reference is made to the Precambrian part of the time scale, which is coming of age in terms of detail, and to the Neogene portion, which has attained an ultra-high-precision absolute-age calibration.
\end{abstract}

\section{Introduction}

The geologic time scale is the framework for deciphering the history of the Earth and has three components:

(1) The international chronostratigraphic divisions and their correlation in the global rock record,

(2) The means of measuring absolute (linear) time or elapsed durations from the rock record, and

(3) The methods of effectively joining the two scales.

For convenience in international communication, the rock record of Earth's history is subdivided in a "chronostratigraphic" scale of standardized global stratigraphic units, such as "Ordovician", "Miocene", "Harpoceras falciferum ammonite Zone" or "polarity Chron C24r". Unlike the continuous ticking clock of the "chronometric" scale (measured in years before present), the chronostratigraphic scale is based on relative time units, in which global reference points at boundary stratotypes define the limits of the main formalized units, such as "Devonian," The chronostratigraphic scale is an agreed convention, whereas its calibration to absolute (linear) time is a matter for discovery or estimation.

By contrast, Precambrian stratigraphy is formally classified chronometrically, i.e. the base of each Precambrian eon, era and period is assigned an arbitrary numerical age. This practice is now being challenged (see below).
Continual improvements in data coverage, methodology, and standardization of chronostratigraphic units imply that no geologic time scale can be final. This brief overview of the status of the Geologic Time Scale in 2004 (GTS2004), documented in detail by Gradstein et al. (2004), is the successor to GTS1989 (Harland et al., 1990), which in turn was preceded by GTS1982 (Harland et al., 1982). GTS2004 also replaces the International Stratigraphic Chart 2000 of the International Commission on Stratigraphy (ICS) and UNESCO, issued four years ago (Remane, 2000).

There are several reasons why this new geologic time scale of 2004 was required, including:

- Nearly 50 of $90+$ Phanerozoic stage boundaries are now defined, versus < 15 in 1990;

- Stable international stage subdivisions rendered invalid about $15 \%$ of the "stage" names of 1990 ;

- Last 23 million years (Neogene) is orbitally tuned with $40 \mathrm{kyr}$ accuracy;

- Orbital scaling has been successful in portions of the Paleocene, lower Cretaceous, lower Jurassic, and upper Triassic;

- Superior stratigraphic integration in Mesozoic has merged direct dating, seafloor spreading (M-sequence), zonal scaling and orbital tuning;

- Superior stratigraphic scaling of Paleozoic was achieved using high-resolution composite zonal standards;

- A 'natural' geologic Precambrian time scale has been proposed to replace the current artificial scale;

- More accurate and precise age dating has provided over 200 $\mathrm{Ar} / \mathrm{Ar}$ and $\mathrm{U} / \mathrm{Pb}$ dates with external (systematic) error analysis, of which only a few of these were available to GTS89;

- Improved mathematical/statistical techniques can combine biostratigraphic zones, polarity chrons, geologic stages and absolute ages to calculate the linear time scale and estimate uncertainty.

A listing is provided at the end of this document of outstanding issues that, once resolved, will pave the way for an updated version of the standard Geologic Time Scale, scheduled under the auspices of ICS for the year 2008 .

\section{Overview of construction of GTS2004}

Since 1989, there have been major developments in time scale research, including:

(1) Stratigraphic standardization through the work of the International Commission on Stratigraphy (ICS) has greatly refined the

Note: This article provides an excerpt of Geologic Time Scale 2004 (Cambridge University Press, 500 pp.). The Time Scale Project is a joint undertaking of F.M. Gradstein, J.G. Ogg, A.G. Smith, F.P. Agterberg, W. Bleeker, R.A. Cooper, V. Davydov, P. Gibbard, L.A. Hinnov, M.R. House ( $\dagger$ ), L.J. Lourens, H-P. Luterbacher, J. McArthur, M.J. Melchin, L.J. Robb, J. Shergold, M. Villeneuve, B.R. Wardlaw, J. Ali, H. Brinkhuis, F.J. Hilgen, J. Hooker, R.J. Howarth, A.H. Knoll, J. Laskar, S. Monechi, J. Powell, K.A. Plumb, I. Raffi, U. Röhl, A. Sanfilippo, B. Schmitz, N.J. Shackleton, G.A. Shields, H. Strauss, J. Van Dam, J. Veizer, Th. van Kolfschoten, and D. Wilson, and is under auspices of the International Commission on Stratigraphy. 
international chronostratigraphic scale. In some cases, such as in the Ordovician or Permian periods, traditional European- or Asianbased geological stages have been replaced with new subdivisions that allow global correlation.

(2) New or enhanced methods of extracting linear time from the rock record have enabled high-precision age assignments. Numerous high-resolution radiometric dates have been generated that has led to improved age assignments of key geologic stage boundaries. The use of global geochemical variations, Milankovitch climate cycles, and magnetic reversals have become important calibration tools.

(3) Statistical techniques of interpolating ages and associated uncertainties to stratigraphic events have evolved to meet the challenge of more accurate age dates and more precise zonal assignments. Fossil event databases with multiple stratigraphic sections through the globe can be integrated into high-resolution composite standards for internal scaling of geologic stages.

The compilation of GTS2004 involved a large number of specialists, listed above, including contributions by past and present chairs of different subcommissions of ICS, geochemists working with radiogenic and stable isotopes, stratigraphers using diverse tools from traditional fossils to astronomical cycles to database programming, and geomathematicians.

The methods used to construct Geologic Time Scale 2004 (GTS2004) integrate different techniques depending on the quality of data available within different intervals (Figure 1). The set of chronostratigraphic units (geologic stages, periods) and their computed ages that constitute the main framework for the Geologic Time Scale 2004 are summarized in the International Stratigraphic Chart (Figure 2 and accompanying insert). Uncertainties on ages are expressed at 2-sigma (95\% confidence). Table 1 summarizes the status of stratigraphic standardization, compiled by one of us (JGO), for the entire geologic column. Steady progress is made with further standardization of the stratigraphic scale.

The main steps involved in the GTS2004 time scale construction were:

Step 1. Construct an updated global chronostratigraphic scale for the Earth's rock record (Table 1).

Step 2. Identify key linear-age calibration levels for the chronostratigraphic scale using radiometric age dates, and/or apply

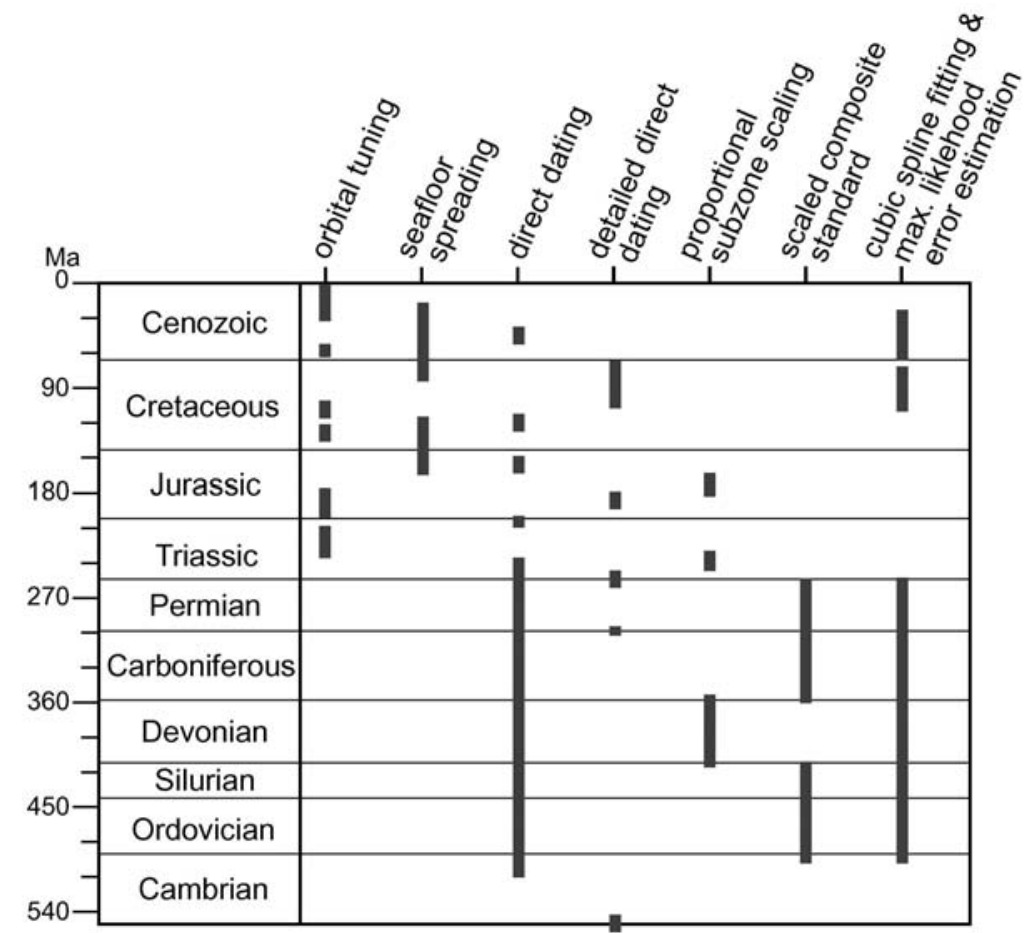

Figure 1 Methods used to construct the Geologic Time Scale 2004 (GTS2004) integrate different techniques depending on the quality of data available within different intervals. astronomical tuning to cyclic sediment or stable isotope sequences which had biostratigraphic or magnetostratigraphic correlations.

Step 3. Interpolate the combined chronostratigraphic and chronometric scale where direct information is insufficient.

Step 4. Calculate or estimate error bars on the combined chronostratigraphic and chronometric information to obtain a geologic time scale with estimates of uncertainty on boundaries and on unit durations.

Step 5. Peer review the geologic time scale through ICS.

The first step, integrating multiple types of stratigraphic information in order to construct the chronostratigraphic scale, is the most time-consuming. This relative geologic time scale summarizes and synthesizes centuries of detailed geological research. The second step, identifying which radiometric and cycle-stratigraphic studies would be used as the primary constraints for assigning linear ages, is the one that is evolved most rapidly during the past decade. Historically, Phanerozoic time scale building went from an exercise with very few and relatively inaccurate radiometric dates, as used by Holmes $(1947,1960)$, to one with many dates with greatly varying analytical precision (like GTS89, or to some extent Gradstein et al., 1994). Next came studies on relatively short stratigraphic intervals that selected a few radiometric dates with high internal analytical precision (e.g., Obradovich, 1993; Cande \& Kent, 1992, 1995; Cooper, 1999) or measured time relative to present using astronomical cycles (e.g., Shackleton et al., 1999; Hilgen et al., 1995, 2000). This later philosophy is adhered to in this scale.

In addition to selecting radiometric ages based upon their stratigraphic control and analytical precision, we also applied the following criteria or corrections:

(1) Stratigraphically constrained radiometric ages with the $\mathrm{U}-\mathrm{Pb}$ method on zircons were accepted from the isotope dilution mass spectrometry (TIMS) method, but generally not from the highresolution ion microprobe (HR-SIMS, also known as "SHRIMP") that uses the Sri Lanka (SL)13 standard. An exception is the Carboniferous Period, where there is a dearth of TIMS dates, and more uncertainty.

(2) ${ }^{40} \mathrm{Ar}-{ }^{39} \mathrm{Ar}$ radiometric ages were re-computed to be in accord with the revised ages for laboratory monitor standards: 523.1 $\pm 4.6 \mathrm{Ma}$ for MMhb-1 (Montana hornblende), $28.34 \pm 0.28 \mathrm{Ma}$ for TCR (Taylor Creek sanidine) and $28.02 \pm 0.28$ Ma for FCT (Fish Canyon sanidine). Systematic ("external") errors and uncertainties in decay constants are partially incorporated. No glauconite dates are used.

The bases of the Paleozoic, Mesozoic and Cenozoic eras are bracketed by analytically precise ages at their GSSP (Global Standard Section and Point) or primary correlation markers $-542 \pm 1.0 \mathrm{Ma}, 251.0 \pm 0.4 \mathrm{Ma}$, and $65.5 \pm 0.3 \mathrm{Ma}$ - and there are direct age-dates on base-Carboniferous, base-Permian, base-Jurassic, and base-Oligocene; but most other period or stage boundaries prior to the Neogene lack direct age control. Therefore, the third step, interpolation, plays a key role for most of GTS2004. A set of detailed and high-resolution interpolation processes incorporated several techniques, depending upon the available information:

(1) A composite standard of graptolite zones spanning the uppermost Cambrian, Ordovician and Silurian interval was derived from $200+$ sections in oceanic and slope environment basins using the constrained optimization method. With zone thickness taken as directly proportional to zone duration, the detailed composite sequence was scaled using selected, high precision zircon and sanidine age dates. For the Carboniferous through Permian a composite standard of conodont, fusulinid, and ammonoids events from many classical sections was calibrated to a combination of U-Pb and ${ }^{40} \mathrm{Ar}-{ }^{39} \mathrm{Ar}$ dates with assigned external error estimates. A composite standard of conodont zones was used for Early Triassic. This procedure directly scaled all stage boundaries and biostratigraphic horizons. 


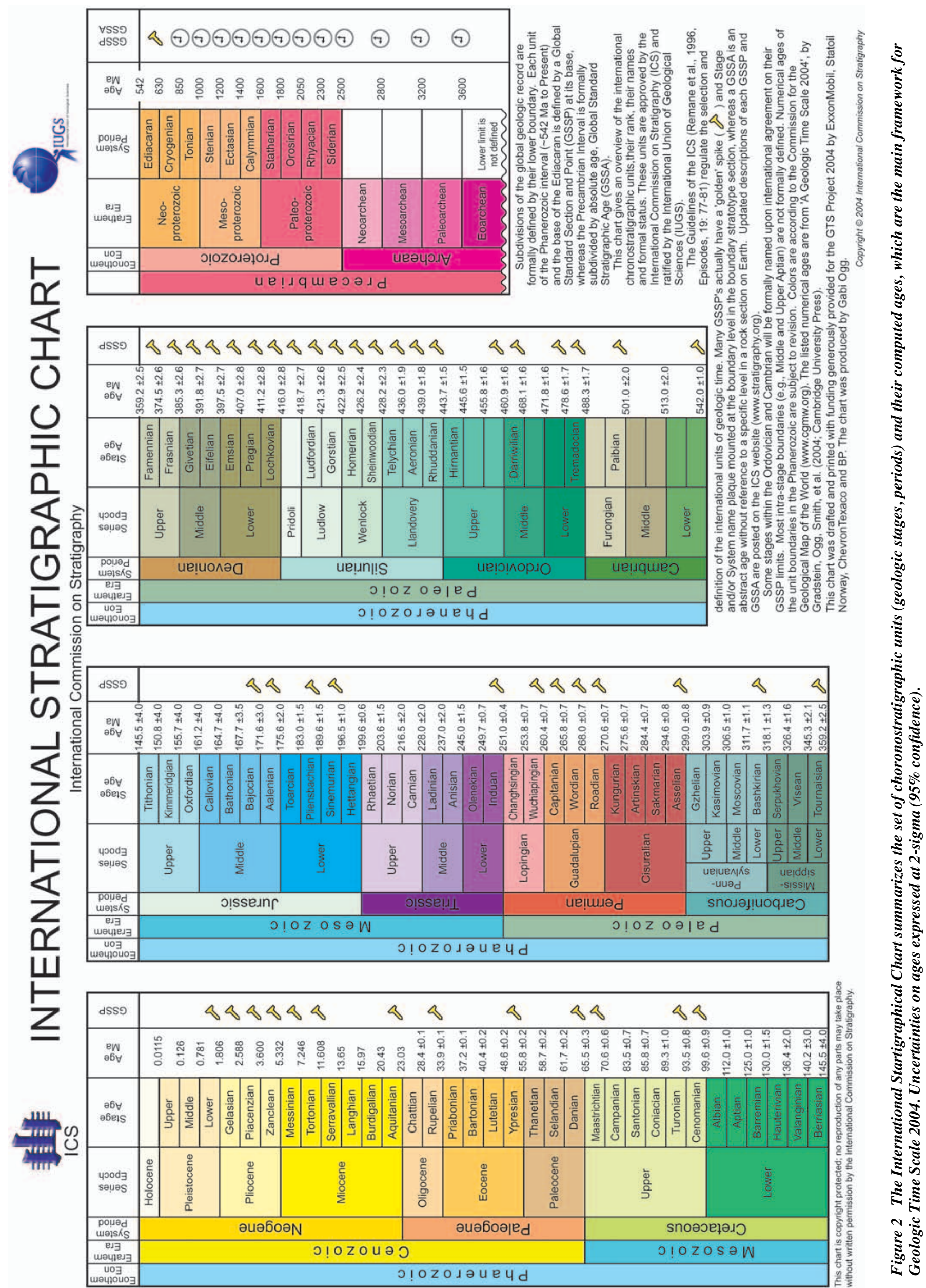


Table 1 Status of defining lower boundaries of geologic stages with GSSPs (as of May, 2004). Updates of this compilation can be obtained from the website (www.stratigraphy.org) of the International Commission on Stratigraphy (ICS) under IUGS.

\begin{tabular}{|c|c|c|c|c|c|c|c|}
\hline $\begin{array}{l}\text { EON, Era, System, } \\
\text { Series, Stage }\end{array}$ & $\begin{array}{l}\text { Age (Ma) } \\
\text { GTS2004 }\end{array}$ & $\begin{array}{c}\text { Est. } \pm \\
\text { myr }\end{array}$ & Derivation of Age & Principal correlative events & GSSP and location & Status & Publication \\
\hline \multicolumn{8}{|l|}{ PHANEROZOIC } \\
\hline \multicolumn{8}{|l|}{ Cenozoic Era } \\
\hline Neogene System & & & & $\begin{array}{l}\text { The "Quaternary" is traditionally considered } \\
\text { to be the interval of oscillating climatic } \\
\text { extremes (glacial and interglacial episodes) } \\
\text { that was initiated at about } 2.6 \mathrm{Ma} \text {, therefore } \\
\text { encompasses the Holocene and Pleistocene } \\
\text { epochs and Gelasian stage of late Pliocene. } \\
\text { This composite epoch is not a formal unit in } \\
\text { the chronostratigraphic hierarchy. }\end{array}$ & & & \\
\hline \multicolumn{8}{|l|}{ Holocene Series } \\
\hline base Holocene & $11.5 \mathrm{ka}$ & 0.00 & $\begin{array}{l}\text { Carbon-14 dating } \\
\text { calibration }\end{array}$ & $\begin{array}{l}\text { exactly } 10,000 \text { Carbon- } 14 \text { years }(=11.5 \mathrm{ka} \\
\text { calendar years BP) at the end of the } \\
\text { Younger Dryas cold spell }\end{array}$ & & $\begin{array}{l}\text { Informal } \\
\text { working } \\
\text { definition }\end{array}$ & \\
\hline \multicolumn{8}{|l|}{ Pleistocene Series } \\
\hline $\begin{array}{l}\text { base Upper Pleistocene } \\
\text { subseries }\end{array}$ & 0.126 & 0.00 & $\begin{array}{l}\text { Astronomical cycles in } \\
\text { sediments }\end{array}$ & $\begin{array}{l}\text { base of the Eemian interglacial stage (= } \\
\text { base of marine isotope stage } 5 e \text { ) before } \\
\text { final glacial episode of Pleistocene }\end{array}$ & $\begin{array}{l}\text { Potentially, within } \\
\text { sediment core under the } \\
\text { Netherlands (Eemian type } \\
\text { area) }\end{array}$ & $\begin{array}{l}\text { Informal } \\
\text { working } \\
\text { definition }\end{array}$ & \\
\hline $\begin{array}{l}\text { base Middle Pleistocene } \\
\text { subseries }\end{array}$ & 0.781 & 0.00 & $\begin{array}{l}\text { Astronomical cycles in } \\
\text { sediments }\end{array}$ & Brunhes-Matuyama magnetic reversal & & $\begin{array}{l}\text { Informal } \\
\text { working } \\
\text { definition }\end{array}$ & \\
\hline base Pleistocene Series & 1.806 & 0.00 & $\begin{array}{l}\text { Astronomical cycles in } \\
\text { sediments }\end{array}$ & $\begin{array}{l}\text { Just above top of magnetic polarity } \\
\text { chronozone C2n (Olduvai) and the } \\
\text { extinction level of calcareous nannofossil } \\
\text { Discoaster brouweri (base Zone } \mathrm{CN} 13 \text { ). } \\
\text { Above are lowest occurrence of calcareous } \\
\text { nannofossil medium Gephyrocapsa spp. } \\
\text { and extinction level of planktonic foraminifer } \\
\text { Globigerinoides extremus. }\end{array}$ & $\begin{array}{l}\text { Top of sapropel layer 'e', } \\
\text { Vrica section, Calabria, } \\
\text { Italy }\end{array}$ & Ratified 1985 & $\begin{array}{l}\text { Episodes } 8(2) \\
\text { p.116-120, } 1985\end{array}$ \\
\hline \multicolumn{8}{|l|}{ Pliocene Series } \\
\hline base Gelasian Stage & 2.588 & 0.00 & $\begin{array}{l}\text { Astronomical cycles in } \\
\text { sediments }\end{array}$ & $\begin{array}{l}\text { Isotopic stage } 103 \text {, base of magnetic } \\
\text { polarity chronozone C2r (Matuyama). } \\
\text { Above are extinction levels of calcareous } \\
\text { nannofossil Discoaster pentaradiatus and } \\
\text { D. surculus (base Zone CN12C). } \\
\end{array}$ & $\begin{array}{l}\text { Midpoint of sapropelic } \\
\text { Nicola Bed ("A5"), Monte } \\
\text { San Nicola, Gela, Sicily, } \\
\text { Italy }\end{array}$ & Ratified 1996 & $\begin{array}{l}\text { Episodes } 21(2) \\
\text { p.82-87, } 1998\end{array}$ \\
\hline base Piacenzian Stage & 3.600 & 0.00 & $\begin{array}{l}\text { Astronomical cycles in } \\
\text { sediments }\end{array}$ & $\begin{array}{l}\text { Base of magnetic polarity chronozone C2An } \\
\text { (Gauss); extinction levels of planktonic } \\
\text { foraminifers Globorotalia margaritae (base } \\
\text { Zone PL3) and Pulleniatina primalis. }\end{array}$ & $\begin{array}{l}\text { Base of beige layer of } \\
\text { carbonate cycle } 77, \text { Punta } \\
\text { Piccola, Sicily, Italy }\end{array}$ & Ratified 1997 & $\begin{array}{l}\text { Episodes } 21(2) \\
\text { p.88-93, } 1998\end{array}$ \\
\hline $\begin{array}{l}\text { base Zanclean Stage, } \\
\text { base Pliocene Series }\end{array}$ & 5.332 & 0.00 & $\begin{array}{l}\text { Astronomical cycles in } \\
\text { sediments }\end{array}$ & $\begin{array}{l}\text { Top of magnetic polarity chronozone C3r, } \\
\text {-100 kyr before Thvera normal-polarity } \\
\text { subchronozone (C } 3 \mathrm{n} .4 \mathrm{n}) \text {. Calcareous } \\
\text { nannofossils - near extinction level of } \\
\text { Triquetrorhabdulus rugosus (base Zone } \\
\text { CN10b) and the lowest occurrence of } \\
\text { Ceratolithus acutus. }\end{array}$ & $\begin{array}{l}\text { Base of Trubi Fm (base of } \\
\text { carbonate cycle 1), } \\
\text { Eraclea Minoa, Sicily, Italy }\end{array}$ & Ratified 2000 & $\begin{array}{l}\text { Episodes } 23(3) \\
\text { p.179-187, } 2000\end{array}$ \\
\hline \multicolumn{8}{|l|}{ Miocene Series } \\
\hline base Messinian Stage & 7.246 & 0.00 & $\begin{array}{l}\text { Astronomical cycles in } \\
\text { sediments }\end{array}$ & $\begin{array}{l}\text { Astrochronology age of } 7.246 \mathrm{Ma} \text {; middle of } \\
\text { magnetic polarity chronozone } \mathrm{C} 3 \mathrm{Br} .1 \mathrm{r} \text {; } \\
\text { lowest regular occurrence of the } \\
\text { Globorotalia conomiozea planktonic } \\
\text { foraminifer group. }\end{array}$ & $\begin{array}{l}\text { Base of red layer of } \\
\text { carbonate cycle } 15, \text { Oued } \\
\text { Akrech, Rabat, Morocco }\end{array}$ & Ratified 2000 & $\begin{array}{l}\text { Episodes } 23(3) \\
\text { p.172-178, } 2000\end{array}$ \\
\hline base Tortonian Stage & 11.608 & 0.00 & $\begin{array}{l}\text { Astronomical cycles in } \\
\text { sediments }\end{array}$ & $\begin{array}{l}\text { Last Common Occurrences of the } \\
\text { calcareous nannofossil Discoaster kugleri } \\
\text { and the planktonic foraminifer } \\
\text { Globigerinoides subquadratus. Associated } \\
\text { with the short normal-polarity subchron } \\
\text { C5r.2n. }\end{array}$ & $\begin{array}{l}\text { Midpoint of sapropel 76, } \\
\text { Monte dei Corvi beach } \\
\text { section, Ancona, Italy }\end{array}$ & Ratified 2003 & $\begin{array}{l}\text { Episodes article in } \\
\text { preparation }\end{array}$ \\
\hline base Serravillian Stage & 13.65 & 0.00 & $\begin{array}{l}\text { Astronomical cycles in } \\
\text { sediments }\end{array}$ & $\begin{array}{l}\text { Near lowest occurrence of nannofossil } \\
\text { Sphenolithus heteromorphus, and within } \\
\text { magnetic polarity chronozone C5ABr. }\end{array}$ & & $\begin{array}{l}\text { GSSP } \\
\text { anticipated in } \\
2004\end{array}$ & \\
\hline base Langhian Stage & 15.97 & 0.0 & $\begin{array}{l}\text { Calibrated magnetic } \\
\text { anomaly scale }\end{array}$ & $\begin{array}{l}\text { Near first occurrence of planktonic } \\
\text { foraminifer Praeorbulina glomerosa and top } \\
\text { of magnetic polarity chronozone C5Cn.1n }\end{array}$ & & \begin{tabular}{|l|} 
GSSP \\
anticipated in \\
2004 \\
\end{tabular} & \\
\hline base Burdigalian Stage & 20.43 & 0.0 & $\begin{array}{l}\text { Calibrated magnetic } \\
\text { anomaly scale }\end{array}$ & $\begin{array}{l}\text { Near lowest occurrence of planktonic } \\
\text { foraminifer Globigerinoides altiaperturus or } \\
\text { near top of magnetic polarity chronozone } \\
\text { C6An }\end{array}$ & & $\begin{array}{l}\text { Guide event } \\
\text { is undecided }\end{array}$ & \\
\hline $\begin{array}{l}\text { base Aquitanian Stage, } \\
\text { base Miocene Series, base } \\
\text { Neogene System }\end{array}$ & 23.03 & 0.0 & $\begin{array}{l}\text { Astronomical cycles in } \\
\text { sediments }\end{array}$ & $\begin{array}{l}\text { Base of magnetic polarity chronozone } \\
\text { C6Cn.2n; lowest occurrence of planktonic } \\
\text { foraminifer Paragloborotalia kugleri; near } \\
\text { extinction of calcareous nannofossil } \\
\text { Reticulofenestra bisecta (base Zone NN1). }\end{array}$ & $\begin{array}{l}35 \mathrm{~m} \text { from top of Lemme- } \\
\text { Carrosio section, Carrosio } \\
\text { village, north of Genoa, } \\
\text { Italy }\end{array}$ & Ratified 1996 & $\begin{array}{l}\text { Episodes } 20(1) \\
\text { p.23-28, } 1997\end{array}$ \\
\hline
\end{tabular}


(Continued)

\begin{tabular}{|c|c|c|c|c|c|c|c|}
\hline $\begin{array}{l}\text { EON, Era, System, } \\
\text { Series, Stage }\end{array}$ & $\begin{array}{l}\text { Age (Ma) } \\
\text { GTS2004 }\end{array}$ & $\begin{array}{l}\text { Est. } \pm \\
\text { myr }\end{array}$ & Derivation of Age & Principal correlative events & GSSP and location & Status & Publication \\
\hline \multicolumn{8}{|l|}{ Paleogene System } \\
\hline \multicolumn{8}{|l|}{ Oligocene Series } \\
\hline base Chattian Stage & 28.4 & 0.1 & $\begin{array}{l}\text { Calibrated magnetic } \\
\text { anomaly scale relative } \\
\text { to base-Miocene and } \\
\text { C24n. Arbitrary } 100 \mathrm{kyr} \\
\text { uncertainty assigned. }\end{array}$ & $\begin{array}{l}\text { Planktonic foraminifer, extinction of } \\
\text { Chiloguembelina (base Zone P21b) }\end{array}$ & $\begin{array}{l}\text { Probably in Umbria- } \\
\text { Marche region of Italy }\end{array}$ & $\begin{array}{l}\text { GSSP } \\
\text { anticipated in } \\
2004\end{array}$ & \\
\hline $\begin{array}{l}\text { base Rupelian Stage, } \\
\text { base Oligocene Series }\end{array}$ & 33.9 & 0.1 & $\begin{array}{l}\text { Calibrated magnetic } \\
\text { anomaly scale relative } \\
\text { to base-Miocene and } \\
\text { C24n. }\end{array}$ & $\begin{array}{l}\text { Planktonic foraminifer, extinction of } \\
\text { Hantkenina }\end{array}$ & $\begin{array}{l}\text { Base of marl bed at } 19 \mathrm{~m} \\
\text { above base of } \\
\text { Massignano quarry, } \\
\text { Ancona, Italy }\end{array}$ & Ratified 1992 & $\begin{array}{l}\text { Episodes } 16(3) \\
\text { p.379-382, } 1993\end{array}$ \\
\hline \multicolumn{8}{|l|}{ Eocene Series } \\
\hline base Priabonian Stage & 37.2 & 0.1 & $\begin{array}{l}\text { Calibrated magnetic } \\
\text { anomaly scale relative } \\
\text { to base-Miocene and } \\
\text { C24n. }\end{array}$ & $\begin{array}{l}\text { Near lowest occurrence of calcareous } \\
\text { nannofossil Chiasmolithus oamaruensis } \\
\text { (base Zone NP18) }\end{array}$ & $\begin{array}{l}\text { Probably in Umbria- } \\
\text { Marche region of Italy }\end{array}$ & & \\
\hline base Bartonian Stage & 40.4 & 0.2 & $\begin{array}{l}\text { Calibrated magnetic } \\
\text { anomaly scale relative } \\
\text { to base-Miocene and } \\
\text { C24n. }\end{array}$ & $\begin{array}{l}\text { Near extinction of calcareous nannofossil } \\
\text { Reticulofenestra reticulata }\end{array}$ & & & \\
\hline base Lutetian Stage & 48.6 & 0.2 & $\begin{array}{l}\text { Calibrated magnetic } \\
\text { anomaly scale relative } \\
\text { to base-Miocene and } \\
\text { C24n. }\end{array}$ & $\begin{array}{l}\text { Planktonic foraminifer, lowest occurrence of } \\
\text { Hantkenina }\end{array}$ & $\begin{array}{l}\text { Leading candidate is } \\
\text { Fortuna section, Murcia } \\
\text { province, Betic } \\
\text { Cordilleras, Spain }\end{array}$ & $\begin{array}{l}\text { GSSP } \\
\text { anticipated in } \\
2004\end{array}$ & \\
\hline $\begin{array}{l}\text { base Ypresian Stage, } \\
\text { base Eocene Series }\end{array}$ & 55.8 & 0.2 & $\begin{array}{l}\text { Astronomical cycles in } \\
\text { sediments scaled from } \\
\text { base-Paleocene }\end{array}$ & Base of negative carbon-isotope excursion & $\begin{array}{l}\text { Dababiya section near } \\
\text { Luxor, Egypt }\end{array}$ & Ratified 2003 & $\begin{array}{l}\text { Micropaleontology } \\
\text { v.49 (Suppl. 1), } \\
\text { 2003. Episodes } \\
\text { article in } \\
\text { preparation }\end{array}$ \\
\hline \multicolumn{8}{|l|}{ Paleocene Series } \\
\hline base Thanetian Stage & 58.7 & 0.2 & $\begin{array}{l}\text { Astronomical cycles in } \\
\text { sediments scaled from } \\
\text { base Paleocene, using } \\
\text { base of magnetic } \\
\text { polarity chronozone } \\
\text { C26n. Arbitrary } 0.1 \text { ( } 2 \\
\text { precession cycles, plus } \\
\text { the base-Paleogene } \\
\text { radiometric) uncertainty } \\
\text { assigned to all } \\
\text { estimates. }\end{array}$ & $\begin{array}{l}\text { Magnetic polarity chronozone, base of } \\
\mathrm{C} 26 \mathrm{n} \text {, is a temporary assignment }\end{array}$ & $\begin{array}{l}\text { Leading candidate is } \\
\text { Zumaya section, northern } \\
\text { Spain }\end{array}$ & $\begin{array}{l}\text { Guide event } \\
\text { is undecided }\end{array}$ & \\
\hline base Selandian Stage & 61.7 & 0.2 & $\begin{array}{l}\text { Astronomical cycles in } \\
\text { sediments scaled from } \\
\text { base Paleocene, using } \\
\text { magnetic polarity } \\
\text { chronozone placement } \\
\text { of } \mathrm{C} 27 \mathrm{n} .9\end{array}$ & $\begin{array}{l}\text { Boundary task group is considering a higher } \\
\text { level - base of calcareous nannofossil zone } \\
\text { NP5 - which would be } \sim 1 \text { myr younger. }\end{array}$ & $\begin{array}{l}\text { Leading candidate is } \\
\text { Zumaya section, northern } \\
\text { Spain }\end{array}$ & $\begin{array}{l}\text { Guide event } \\
\text { is undecided }\end{array}$ & \\
\hline $\begin{array}{l}\text { base Danian Stage, base } \\
\text { Paleogene System, base } \\
\text { Cenozoic }\end{array}$ & 65.5 & 0.3 & $\begin{array}{l}\text { Ar-Ar and U-Pb age } \\
\text { agreement }\end{array}$ & $\begin{array}{l}\text { Iridium geochemical anomaly. Associated } \\
\text { with a major extinction horizon } \\
\text { (foraminifers, calcareous nannofossils, } \\
\text { dinosaurs, etc.); }\end{array}$ & $\begin{array}{l}\text { Base of boundary clay, El } \\
\text { Kef, Tunisia (but } \\
\text { deterioration may require } \\
\text { assigning a replacement } \\
\text { section) }\end{array}$ & Ratified 1991 & \\
\hline \multicolumn{8}{|l|}{ Mesozoic Era } \\
\hline Cretaceous System & & & & $\begin{array}{l}\text { Most substages of Cretaceous also have } \\
\text { recommended GSSP criteria }\end{array}$ & & & \\
\hline \multicolumn{8}{|l|}{ Upper } \\
\hline $\begin{array}{l}\text { base Maastrichtian } \\
\text { Stage }\end{array}$ & 70.6 & 0.6 & $\begin{array}{l}\text { Estimated placement } \\
\text { relative to Ar-Ar } \\
\text { calibrated Sr-curve }\end{array}$ & $\begin{array}{l}\text { Mean of } 12 \text { biostratigraphic criteria of equal } \\
\text { importance. Closely above is lowest } \\
\text { occurrence of ammonite Pachydiscus } \\
\text { neubergicus. Boreal proxy is lowest } \\
\text { occurrence of belemnite Belemnella } \\
\text { lanceolata. }\end{array}$ & $\begin{array}{l}115.2 \text { m level in Grande } \\
\text { Carrière quarry, Tercis- } \\
\text { les-Bains, Landes } \\
\text { province, SW France }\end{array}$ & Ratified 2001 & $\begin{array}{l}\text { Episodes } 24 \text { (4), } \\
\text { p.229-238, 2001; } \\
\text { Odin (ed.) IUGS } \\
\text { Spec. Publ. } \\
\text { Series, v.36, } \\
\text { Elsevier, 910pp. }\end{array}$ \\
\hline base Campanian Stage & 83.5 & 0.7 & $\begin{array}{l}\text { Spline fit of } \mathrm{Ar}-\mathrm{Ar} \text { ages } \\
\text { and ammonite zones. }\end{array}$ & $\begin{array}{l}\text { Crinoid, extinction of Marsupites } \\
\text { testudinarius }\end{array}$ & $\begin{array}{l}\text { Leading candidates are in } \\
\text { southern England and in } \\
\text { Texas }\end{array}$ & & \\
\hline base Santonian Stage & 85.9 & 0.7 & $\begin{array}{l}\text { Spline fit of Ar-Ar ages } \\
\text { and ammonite zones. }\end{array}$ & $\begin{array}{l}\text { Inoceramid bivalve, lowest occurrence of } \\
\text { Cladoceramus undulatoplicatus }\end{array}$ & $\begin{array}{l}\text { Leading candidates are in } \\
\text { Spain, England and Texas }\end{array}$ & & \\
\hline base Coniacian Stage & 89.3 & 1.0 & $\begin{array}{l}\text { Spline fit of Ar-Ar ages } \\
\text { and ammonite zones. }\end{array}$ & $\begin{array}{l}\text { Inoceramid bivalve, lowest occurrence of } \\
\text { Cremnoceramus rotundatus (sensu Tröger } \\
\text { non Fiege) }\end{array}$ & $\begin{array}{l}\text { Base of Bed MK47, } \\
\text { Salzgitter-Salder Quarry, } \\
\text { SW of Hannover, Lower } \\
\text { Saxony, northern } \\
\text { Germany }\end{array}$ & $\begin{array}{l}\text { GSSP } \\
\text { anticipated in } \\
2004\end{array}$ & \\
\hline
\end{tabular}


(Continued)

\begin{tabular}{|c|c|c|c|c|c|c|c|}
\hline $\begin{array}{l}\text { EON, Era, System, } \\
\text { Series, Stage }\end{array}$ & $\begin{array}{l}\text { Age (Ma) } \\
\text { GTS2004 }\end{array}$ & $\begin{array}{c}\text { Est. } \pm \\
\text { myr }\end{array}$ & Derivation of Age & Principal correlative events & GSSP and location & Status & Publication \\
\hline base Turonian Stage & 93.6 & 0.8 & $\begin{array}{l}\text { Spline fit of Ar-Ar ages } \\
\text { and ammonite zones. }\end{array}$ & $\begin{array}{l}\text { Ammonite, lowest occurrence of } \\
\text { Watinoceras devonense }\end{array}$ & $\begin{array}{l}\text { Base of Bed } 86, \text { Rock } \\
\text { Canyon Anticline, east of } \\
\text { Pueblo, Colorado, west- } \\
\text { central USA }\end{array}$ & Ratified 2003 & $\begin{array}{l}\text { Episodes article in } \\
\text { preparation }\end{array}$ \\
\hline base Cenomanian Stage & 99.6 & 0.9 & $\begin{array}{l}\text { Spline fit of Ar-Ar ages } \\
\text { and ammonite zones, } \\
\text { plus monitor standard } \\
\text { correction. Then cycle } \\
\text { stratigraphy to place } \\
\text { foraminifer datum } \\
\text { relative to ammonite } \\
\text { zonation. } \\
\end{array}$ & $\begin{array}{l}\text { Planktonic foraminifer, lowest occurrence of } \\
\text { Rotalipora globotruncanoides }\end{array}$ & $\begin{array}{l}36 \mathrm{~m} \text { below top of Marnes } \\
\text { Bleues Formation, Mont } \\
\text { Risou, Rosans, Haute- } \\
\text { Alpes, SE France }\end{array}$ & Ratified 2002 & $\begin{array}{l}\text { Episodes } 27(1) \\
\text { p.21-32, } 2004\end{array}$ \\
\hline \multicolumn{8}{|l|}{ Lower } \\
\hline base Albian Stage & 112.0 & 1.0 & $\begin{array}{l}\text { Estimated placement } \\
\text { relative to bases of } \\
\text { Cenomanian and } \\
\text { Aptian, with large } \\
\text { uncertainty due to lack } \\
\text { of GSSP criteria. Ar-Ar } \\
\text { age of } 114.6+1-0.7 \mathrm{Ma} \\
\text { from Parahoplites } \\
\text { nutfieldensis below. }\end{array}$ & $\begin{array}{l}\text { Calcareous nannofossil, lowest occurrence } \\
\text { of Praediscosphaera columnata ( } P \text {. } \\
\text { cretacea of some earlier studies), is one } \\
\text { potential marker. }\end{array}$ & & $\begin{array}{l}\text { Guide event } \\
\text { is undecided }\end{array}$ & \\
\hline base Aptian Stage & 125.0 & 1.0 & $\begin{array}{l}\text { Base of MOr, as } \\
\text { recomputed from Ar-Ar } \\
\text { age from MIT guyot }\end{array}$ & Magnetic polarity chronozone, base of MOr & $\begin{array}{l}\text { Leading candidate is } \\
\text { Gorgo a Cerbara, } \\
\text { Piobbico, Umbria-Marche, } \\
\text { central Italy }\end{array}$ & & \\
\hline base Barremian Stage & 130.0 & 1.5 & $\begin{array}{l}\text { Pacific spreading model } \\
\text { for magnetic anomaly } \\
\text { ages (variable rate), } \\
\text { using placement at } \\
\text { M5n.8. }\end{array}$ & $\begin{array}{l}\text { Ammonite, lowest occurrence of Spitidiscus } \\
\text { hugii - Spitidiscus vandeckii group }\end{array}$ & $\begin{array}{l}\text { Leading candidate is Rio } \\
\text { Argos near Caravaca, } \\
\text { Murcia province, Spain }\end{array}$ & & \\
\hline base Hauterivian Stage & 136.4 & 2.0 & $\begin{array}{l}\text { Pacific spreading model } \\
\text { for magnetic anomaly } \\
\text { ages(variable rate),using } \\
\text { placement at base M11n. }\end{array}$ & $\begin{array}{l}\text { Ammonite, lowest occurrence of genus } \\
\text { Acanthodiscus (especially A. radiatus) }\end{array}$ & $\begin{array}{l}\text { Leading candidate is La } \\
\text { Charce village, Drôme } \\
\text { province, southeast } \\
\text { France }\end{array}$ & & \\
\hline base Valanginian Stage & 140.2 & 3.0 & $\begin{array}{l}\text { Pacific spreading model } \\
\text { for magnetic anomaly } \\
\text { ages (variable rate), } \\
\text { using placement at } \\
\text { M14r.3 (base } T \text {. } \\
\text { pertransiens). }\end{array}$ & $\begin{array}{l}\text { Calpionellid, lowest occurrence of } \\
\text { Calpionellites darderi (base of Calpionellid } \\
\text { Zone E); followed by the lowest occurrence } \\
\text { of ammonite "Thurmanniceras" } \\
\text { pertransiens }\end{array}$ & $\begin{array}{l}\text { Leading candidate is near } \\
\text { Montbrun-les-Bains, Drô } \\
\text { me province, southeast } \\
\text { France }\end{array}$ & & \\
\hline $\begin{array}{l}\text { base Berriasian Stage, } \\
\text { base Cretaceous System }\end{array}$ & 145.5 & 4.0 & $\begin{array}{l}\text { Pacific spreading model } \\
\text { for magnetic anomaly } \\
\text { ages (variable rate), } \\
\text { assigning to base of } \\
\text { Berriasella jacobi zone } \\
\text { (M19n.2n.55) }\end{array}$ & $\begin{array}{l}\text { Maybe near lowest occurrence of ammonite } \\
\text { Berriasella jacobi }\end{array}$ & & $\begin{array}{l}\text { Guide event } \\
\text { is undecided }\end{array}$ & \\
\hline \multicolumn{8}{|l|}{ Jurassic System } \\
\hline \multicolumn{8}{|l|}{ Upper } \\
\hline base Tithonian Stage & 150.8 & 4.0 & $\begin{array}{l}\text { Pacific spreading model } \\
\text { for magnetic anomaly } \\
\text { ages (variable rate), } \\
\text { assigning to base } \\
\text { M22An }\end{array}$ & $\begin{array}{l}\text { Near base of Hybonoticeras hybonotum } \\
\text { ammonite zone and lowest occurrence of } \\
\text { Gravesia genus, and the base of magnetic } \\
\text { polarity chronozone M22An }\end{array}$ & & $\begin{array}{l}\text { Guide event } \\
\text { is undecided }\end{array}$ & \\
\hline $\begin{array}{l}\text { base Kimmeridgian } \\
\text { Stage }\end{array}$ & 155.7 & 4.0 & $\begin{array}{l}\text { Pacific spreading model } \\
\text { for magnetic anomaly } \\
\text { ages (variable rate), } \\
\text { assigning to base } \\
\text { M26r.2 (Boreal } \\
\text { ammonite definition) }\end{array}$ & $\begin{array}{l}\text { Ammonite, near base of Pictonia baylei } \\
\text { ammonite zone of Boreal realm }\end{array}$ & $\begin{array}{l}\text { Leading candidates are In } \\
\text { Scotland, SE France and } \\
\text { Poland }\end{array}$ & $\begin{array}{l}\text { GSSP } \\
\text { anticipated in } \\
2004\end{array}$ & \\
\hline base Oxfordian Stage & 161.2 & 4.0 & $\begin{array}{l}\text { Pacific spreading model } \\
\text { for magnetic anomaly } \\
\text { ages (variable rate), } \\
\text { assigning to base } \\
\text { M36An }\end{array}$ & $\begin{array}{l}\text { Ammonite, Brightia thuouxensis Horizon at } \\
\text { base of the Cardioceras scarburgense } \\
\text { Subzone (Quenstedtoceras mariae Zone) }\end{array}$ & $\begin{array}{l}\text { Leading candidates are in } \\
\text { SE France and southern } \\
\text { England }\end{array}$ & $\begin{array}{l}\text { GSSP } \\
\text { anticipated in } \\
2004\end{array}$ & \\
\hline \multicolumn{8}{|l|}{ Middle } \\
\hline base Callovian Stage & 164.7 & 4.0 & $\begin{array}{l}\text { Equal subzones scale } \\
\text { Bajo-Bath-Callov }\end{array}$ & $\begin{array}{l}\text { Ammonite, lowest occurrence of the genus } \\
\text { Kepplerites (Kosmoceratidae) (defines } \\
\text { base of Macrocephalites herveyi Zone in } \\
\text { sub-Boreal province of Great Britain to } \\
\text { southwest Germany) }\end{array}$ & $\begin{array}{l}\text { Leading candidate is } \\
\text { Pfeffingen, Swabian Alb, } \\
\text { SW Germany }\end{array}$ & $\begin{array}{l}\text { GSSP } \\
\text { anticipated in } \\
2004\end{array}$ & \\
\hline base Bathonian Stage & 167.7 & 3.5 & $\begin{array}{l}\text { Equal subzones scale } \\
\text { Bajo-Bath-Callov }\end{array}$ & $\begin{array}{l}\text { Ammonite, lowest occurrence of } \\
\text { Parkinsonia (G.) convergens (defines base } \\
\text { of Zigzagiceras zigzag Zone) }\end{array}$ & & & \\
\hline
\end{tabular}


(Continued)

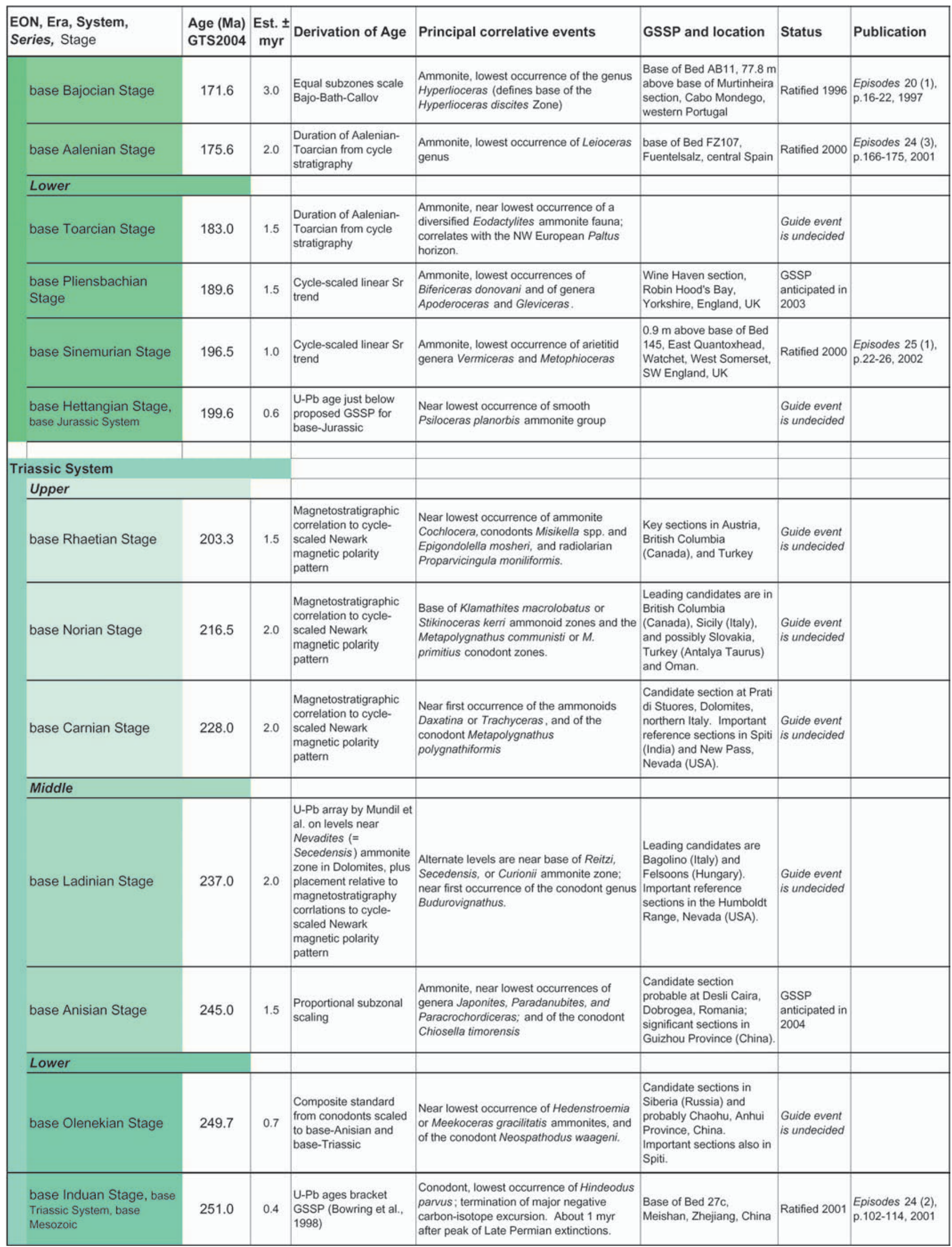


(Continued)

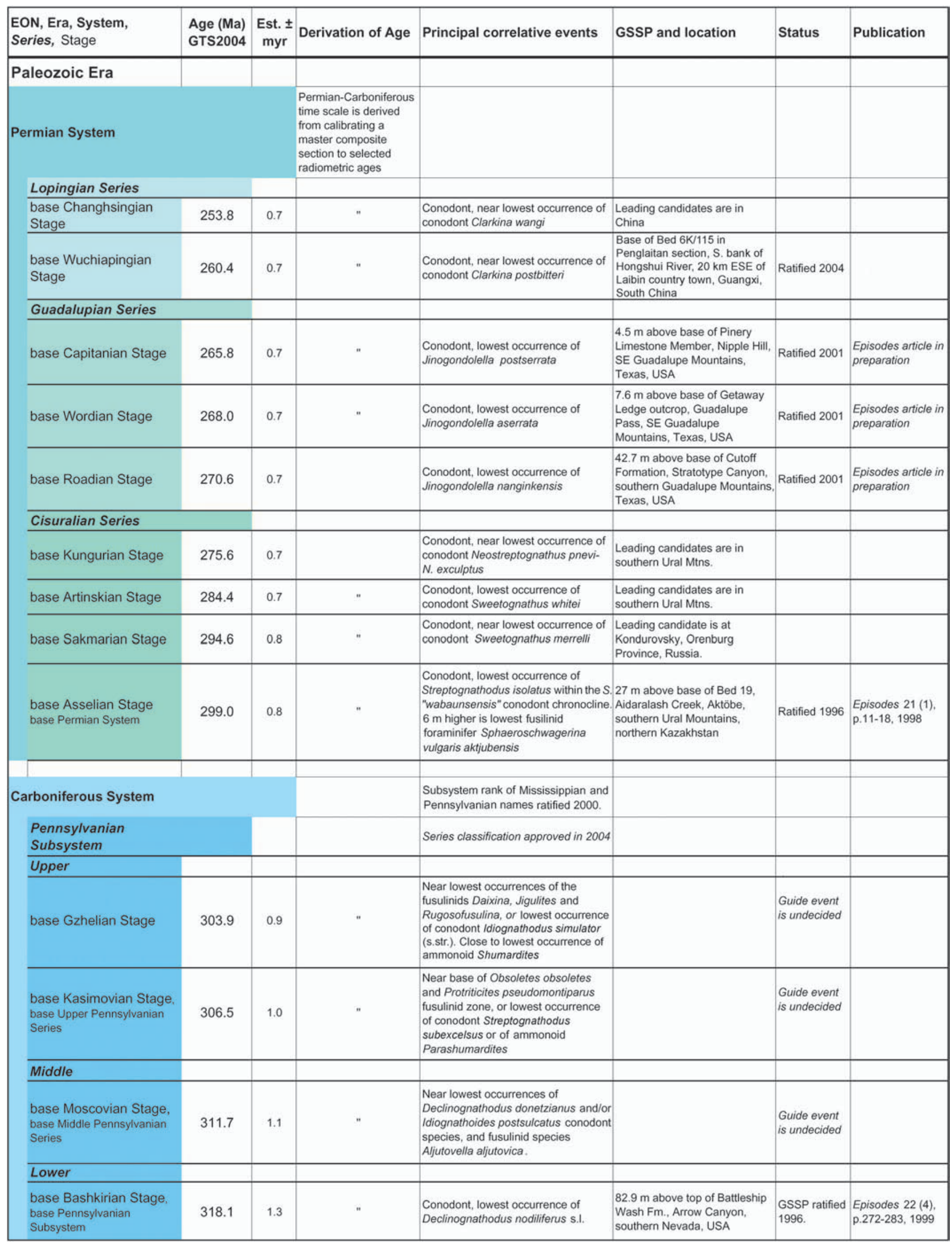


(Continued)

\begin{tabular}{|c|c|c|c|c|c|c|c|}
\hline $\begin{array}{l}\text { EON, Era, System, } \\
\text { Series, Stage }\end{array}$ & $\begin{array}{l}\text { Age (Ma) } \\
\text { GTS2004 }\end{array}$ & $\begin{array}{c}\text { Est. } \pm \\
\text { myr }\end{array}$ & Derivation of Age & Principal correlative events & GSSP and location & Status & Publication \\
\hline \multicolumn{2}{|l|}{$\begin{array}{l}\text { Mississippian } \\
\text { Subsystem }\end{array}$} & & & Series classification approved in 2004 & & & \\
\hline $\begin{array}{l}\text { base Serpukhovian, base } \\
\text { Upper Mississippian Series }\end{array}$ & 326.4 & 1.6 & " & $\begin{array}{l}\text { Near lowest occurrence of conodont, } \\
\text { Lochriea crusiformis. }\end{array}$ & & $\begin{array}{l}\text { Guide event } \\
\text { is undecided }\end{array}$ & \\
\hline $\begin{array}{l}\text { base Visean, base Middle } \\
\text { Mississippian Series }\end{array}$ & 345.3 & 2.1 & $"$ & $\begin{array}{l}\text { Foraminifer, lineage Eoparastaffella } \\
\text { simplex morphotype } 1 / \text { morphotype } 2\end{array}$ & $\begin{array}{l}\text { Leading candidate is } \\
\text { Pengchong, south China }\end{array}$ & & \\
\hline $\begin{array}{l}\text { base Tournaisian, base } \\
\text { Mississippian Subsystem, } \\
\text { base Carboniferous System }\end{array}$ & 359.2 & 2.5 & " & $\begin{array}{l}\text { Conodont, above lowest occurrence } \\
\text { of Siphonodella sulcata }\end{array}$ & $\begin{array}{l}\text { Base of Bed } 89 \text {, La Serre, } \\
\text { Montagne Noir, Cabrières, } \\
\text { southern France }\end{array}$ & Ratified 1990 & $\begin{array}{l}\text { Episodes } 14(4), \\
\text { p.331-336, } 1991\end{array}$ \\
\hline \multirow{2}{*}{\multicolumn{2}{|c|}{ Devonian System }} & & $\begin{array}{l}\text { Devonian time scale is } \\
\text { a statistical fit of a } \\
\text { composite } \\
\text { biostratigraphic } \\
\text { zonation (based on } \\
\text { Figure } 8 \text { of Williams et } \\
\text { al., } 2000 \text { ) to selected } \\
\text { radiometric ages }\end{array}$ & & & & \\
\hline & & \multicolumn{6}{|c|}{ Upper } \\
\hline base Famennian Stage & 374.5 & 2.6 & " & $\begin{array}{l}\text { Just above major extinction horizon } \\
\text { (Upper Kellwasser Event), including } \\
\text { conodonts Ancyrodella and } \\
\text { Ozarkodina and goniatites of } \\
\text { Gephuroceratidae and Beloceratidae }\end{array}$ & $\begin{array}{l}\text { base of Bed 32a, upper } \\
\text { Coumiac quarry, Cessenon, } \\
\text { Montagne Noir, southern } \\
\text { France }\end{array}$ & Ratified 1993 & $\begin{array}{l}\text { Episodes } 16(4), \\
\text { p.433-441, } 1993\end{array}$ \\
\hline base Frasnian Stage & 385.3 & 2.6 & " & $\begin{array}{l}\text { Conodont, lowest occurrence of } \\
\text { Ancyrodella rotundiloba (defines base } \\
\text { of Lower Polygnathus asymmetricus } \\
\text { conodont Zone) }\end{array}$ & $\begin{array}{l}\text { Base of Bed 42a', Col du Puech } \\
\text { de la Suque section, St. } \\
\text { Nazaire-de-Ladarez, SE } \\
\text { Montagne Noir, southern } \\
\text { France }\end{array}$ & Ratified 1986 & $\begin{array}{l}\text { Episodes } 10(2) \\
\text { p. } 97-101,1987\end{array}$ \\
\hline \multicolumn{8}{|l|}{ Middle } \\
\hline base Givetian Stage & 391.8 & 2.7 & " & $\begin{array}{l}\text { Conodont, lowest occurrence of } \\
\text { Polygnathus hemiansatus, near base } \\
\text { of goniatite Maenioceras Stufe }\end{array}$ & $\begin{array}{l}\text { Base of Bed } 123 \text {, Jebel Mech } \\
\text { Irdane ridge, Tafilalt, Morocco }\end{array}$ & Ratified 1994 & $\begin{array}{l}\text { Episodes } 18(3), \\
\text { p.107-115, } 1995\end{array}$ \\
\hline base Eifelian Stage & 397.5 & 2.7 & " & $\begin{array}{l}\text { Conodont, lowest occurrence of } \\
\text { Polygnathus costatus partitus; major } \\
\text { faunal turnover }\end{array}$ & $\begin{array}{l}\text { Base unit WP } 30 \text {, trench at } \\
\text { Wetteldorf Richtschnitt, Schö } \\
\text { necken-Wetteldorf, Eifel Hills, } \\
\text { western Germany }\end{array}$ & Ratified 1985 & $\begin{array}{l}\text { Episodes } 8(2) \\
\text { p.104-109, } 1985\end{array}$ \\
\hline \multicolumn{8}{|l|}{ Lower } \\
\hline base Emsian Stage & 407.0 & 2.8 & " & $\begin{array}{l}\text { Conodont, lowest occurrence of } \\
\text { Polygnathus kitabicus (= Po. } \\
\text { dehiscens) }\end{array}$ & $\begin{array}{l}\text { Base of Bed 9/5, Zinzil'ban } \\
\text { Gorge, SE of Samarkand, } \\
\text { Uzbekistan }\end{array}$ & Ratified 1995 & $\begin{array}{l}\text { Episodes } 20 \text { (4), } \\
\text { p.235-240, } 1997\end{array}$ \\
\hline base Pragian Stage & 411.2 & 2.8 & " & $\begin{array}{l}\text { Conodont, lowest occurrence of } \\
\text { Eognathodus sulcatus }\end{array}$ & $\begin{array}{l}\text { Base of Bed 12, Velka Chuchle } \\
\text { quarry, southwest part of } \\
\text { Prague city, Czech Republic }\end{array}$ & Ratified 1989 & $\begin{array}{l}\text { Episodes } 12(2) \\
\text { p. } 109-113,1989\end{array}$ \\
\hline $\begin{array}{l}\text { base Lochkovian Stage, } \\
\text { base Devonian System }\end{array}$ & 416.0 & 2.8 & $\begin{array}{l}\text { base-Devonian from } \\
\text { scale in Cooper (this } \\
\text { volume), which is } 1 \mathrm{myr} \\
\text { younger than Tucker et } \\
\text { al (1998) estimate. }\end{array}$ & $\begin{array}{l}\text { Graptolite, lowest occurrence of } \\
\text { Monograptus uniformis }\end{array}$ & $\begin{array}{l}\text { Within Bed 20, Klonk, } \\
\text { Barrandian area, southwest of } \\
\text { Prague, Czech Republic }\end{array}$ & Ratified 1972 & $\begin{array}{l}\text { Martinsson (ed.), } \\
\text { The Silurian- } \\
\text { Devonian } \\
\text { Boundary, IUGS } \\
\text { Series A, no.5. } \\
349 \text { pp., } 1977\end{array}$ \\
\hline \multirow{2}{*}{\multicolumn{2}{|c|}{ Silurian System }} & & $\begin{array}{l}\text { Silurian and Ordovician } \\
\text { time scales are from } \\
\text { calibrating a CONOP } \\
\text { composite graptolite } \\
\text { zonation to selecte } \\
\text { radiometric ages }\end{array}$ & & & & $\begin{array}{l}\text { Holland and } \\
\text { Bassett (eds), A } \\
\text { Global Standard } \\
\text { for the Silurian } \\
\text { System, Nat. Mus. } \\
\text { Wales, Geol. } \\
\text { Series No.10, } \\
\text { Cardiff, } 325 \text { pp., } \\
1989\end{array}$ \\
\hline & & & & & & & \\
\hline $\begin{array}{l}\text { base Pridoli Series (not } \\
\text { subdivided in stages) }\end{array}$ & 418.7 & 2.7 & " & $\begin{array}{l}\text { Graptolite, lowest occurrence of } \\
\text { Monograptus parultimus }\end{array}$ & $\begin{array}{l}\text { Within Bed 96, Pozáry section } \\
\text { near Reporje, Barrandian area, } \\
\text { Prague, Czech Republic }\end{array}$ & Ratified 1984 & $\begin{array}{l}\text { Episodes } 8(2), \\
\text { p.101-103, 1985 }\end{array}$ \\
\hline \multicolumn{2}{|l|}{ Ludlow Series } & 2.6 & & & & & \\
\hline base Ludfordian Stage & 421.3 & 2.6 & " & $\begin{array}{l}\text { Imprecise. May be near base of } \\
\text { Saetograptus leintwardinensis } \\
\text { graptolite zone. }\end{array}$ & $\begin{array}{l}\text { Base of lithological unit C, } \\
\text { Sunnyhill Quarry, Ludlow, } \\
\text { Shropshire, southwest England, } \\
\text { UK }\end{array}$ & Ratified 1980 & $\begin{array}{l}\text { Lethaia 14, p.168, } \\
\text { 1981; Episodes } 5 \\
\text { (3), p.21-23, } 1982\end{array}$ \\
\hline
\end{tabular}


(Continued)

\begin{tabular}{|c|c|c|c|c|c|c|c|}
\hline $\begin{array}{l}\text { EON, Era, System, } \\
\text { Series, Stage }\end{array}$ & $\begin{array}{l}\text { Age (Ma) } \\
\text { GTS2004 }\end{array}$ & $\begin{array}{c}\text { Est. } \pm \\
\text { myr }\end{array}$ & Derivation of Age & Principal correlative events & GSSP and location & Status & Publication \\
\hline base Gorstian Stage & 422.9 & 2.5 & $"$ & $\begin{array}{l}\text { Imprecise. Just below base of local } \\
\text { acritarch Leptobrachion longhopense } \\
\text { range zone. May be near base of } \\
\text { Neodiversograptus nilssoni graptolite } \\
\text { zone. }\end{array}$ & $\begin{array}{l}\text { Base of lithological unit F, Pitch } \\
\text { Coppice quarry, Ludlow, } \\
\text { Shropshire, southwest England, } \\
\text { UK }\end{array}$ & Ratified 1980 & $\begin{array}{l}\text { Lethaia 14, p.168, } \\
\text { 1981; Episodes } 5 \\
\text { (3), p.21-23, } 1982\end{array}$ \\
\hline \multicolumn{8}{|l|}{ Wenlock Series } \\
\hline $\begin{array}{l}\text { base Sheinwoodian } \\
\text { Stage }\end{array}$ & 428.2 & 2.3 & " & $\begin{array}{l}\text { Imprecise. Between the base of } \\
\text { acritarch biozone } 5 \text { and extinction of } \\
\text { conodont Pterospathodus } \\
\text { amorphognathoides. May be near } \\
\text { base of Cyrtograptus centrifugus } \\
\text { graptolite zone. }\end{array}$ & $\begin{array}{l}\text { Base of lithological unit G, } \\
\text { Hughley Brook, Apedale, } \\
\text { Shropshire, southwest England, } \\
\text { UK }\end{array}$ & Ratified 1980 & $\begin{array}{l}\text { Lethaia 14, p.168, } \\
\text { 1981; Episodes } 5 \\
\text { (3), p.21-23, } 1982\end{array}$ \\
\hline \multicolumn{8}{|l|}{ Llandovery Series } \\
\hline base Telychian Stage & 436.1 & 1.9 & $"$ & $\begin{array}{l}\text { Brachiopods, just above extinction of } \\
\text { Eocoelia intermedia and below lowest } \\
\text { succeeding species Eocoelia curtisi. } \\
\text { Near base of Monograptus } \\
\text { turniculatus graptolite zone. }\end{array}$ & $\begin{array}{l}\text { Locality } 162 \text { in transect d, Cefn } \\
\text { Cerig road, Llandovery area, } \\
\text { south-central Wales, UK }\end{array}$ & Ratified 1984 & $\begin{array}{l}\text { Episodes 8 (2), } \\
\text { p.101-103, } 1985\end{array}$ \\
\hline base Aeronian Stage & 439.0 & 1.8 & $"$ & $\begin{array}{l}\text { Graptolite, lowest occurrence of } \\
\text { Monograptus austerus sequens } \\
\text { (defines base of Monograptus } \\
\text { triangulatus graptolite zone) }\end{array}$ & $\begin{array}{l}\text { Base of locality } 72 \text { in transect } h \text {, } \\
\text { Trefawr forestry road, north of } \\
\text { Cwm-coed-Aeron Farm, } \\
\text { Llandovery area, south-central } \\
\text { Wales, UK }\end{array}$ & Ratified 1984 & $\begin{array}{l}\text { Episodes 8 (2), } \\
\text { p.101-103, } 1985\end{array}$ \\
\hline $\begin{array}{l}\text { base Rhuddanian Stage, } \\
\text { base Silurian System }\end{array}$ & 443.7 & 1.5 & $"$ & $\begin{array}{l}\text { Graptolites, lowest occurrences of } \\
\text { Parakidograptus acuminatus and } \\
\text { Akidograptus ascensus }\end{array}$ & $\begin{array}{l}1.6 \mathrm{~m} \text { above base of Birkhill } \\
\text { Shale Fm., Dob's Linn, Moffat, } \\
\text { Scotland, UK }\end{array}$ & Ratified 1984 & $\begin{array}{l}\text { Episodes } 8(2) \\
\text { p.98-100, } 1985\end{array}$ \\
\hline & & & & & & & \\
\hline \multicolumn{8}{|l|}{ Ordovician System } \\
\hline \multicolumn{8}{|l|}{ Upper } \\
\hline base Hirnantian Stage & 445.6 & 1.5 & $"$ & $\begin{array}{l}\text { Potentially at base of the } \\
\text { Normalograptus extraordinarius- } N \text {. } \\
\text { ojsuensis graptolite biozone }\end{array}$ & $\begin{array}{l}\text { Candidate section is } \\
\text { Wangjiawan, China }\end{array}$ & & \\
\hline $\begin{array}{l}\text { base of fifth stage (not yet } \\
\text { named) }\end{array}$ & 460.9 & 1.6 & $"$ & $\begin{array}{l}\text { Graptolite, lowest occurrence of } \\
\text { Nemagraptus gracilis }\end{array}$ & $\begin{array}{l}1.4 \mathrm{~m} \text { below phosphorite in } \\
\text { E14a outcrop, Fágelsáng, } \\
\text { Scane, southern Sweden }\end{array}$ & Ratified 2002 & \begin{tabular}{|l|} 
Episodes $23(2)$, \\
p.102-109, 2000 \\
(proposal; formal \\
GSSP publication \\
in preparation). \\
\end{tabular} \\
\hline \multicolumn{8}{|l|}{ Middle } \\
\hline base Darriwilian Stage & 468.1 & 1.6 & $"$ & $\begin{array}{l}\text { Graptolite, lowest occurrence of } \\
\text { Undulograptus austrodentatus }\end{array}$ & $\begin{array}{l}\text { Base of Bed AEP184, } 22 \mathrm{~m} \\
\text { below top of Ningkuo Fm., } \\
\text { Huangnitang, Changshan, } \\
\text { Zhejiang province, southeast } \\
\text { China }\end{array}$ & Ratified 1997 & $\begin{array}{l}\text { Episodes } 20(3), \\
\text { p.158-166, } 1997\end{array}$ \\
\hline $\begin{array}{l}\text { base of third stage (not yet } \\
\text { named) }\end{array}$ & 471.8 & 1.6 & $"$ & $\begin{array}{l}\text { Conodont, potentially lowest } \\
\text { occurrence of Protoprioniodus aranda } \\
\text { or of Baltoniodus triangularis }\end{array}$ & $\begin{array}{l}\text { Candidate sections at Niquivil } \\
\text { (Argentina) and } \\
\text { Huanghuachang (China) }\end{array}$ & & \\
\hline \multicolumn{8}{|l|}{ Lower } \\
\hline $\begin{array}{l}\text { base of second stage (not yet } \\
\text { named) }\end{array}$ & 478.6 & 1.7 & $"$ & $\begin{array}{l}\text { Graptolite, lowest occurrence of } \\
\text { Tetragraptus approximatus }\end{array}$ & \begin{tabular}{|l|} 
Just above E bed, \\
Diabasbrottet quarry, Văstergö \\
tland, southern Sweden
\end{tabular} & Ratified 2002 & $\begin{array}{l}\text { Episodes article in } \\
\text { preparation }\end{array}$ \\
\hline $\begin{array}{l}\text { base of Tremadocian } \\
\text { Stage, base Ordovician } \\
\text { System }\end{array}$ & 488.3 & 1.7 & $"$ & $\begin{array}{l}\text { Conodont, lowest occurrence of } \\
\text { lapetognathus fluctivagus; just above } \\
\text { base of Cordylodus lindstromi } \\
\text { conodont Zone. Just below lowest } \\
\text { occurrence of planktonic graptolites. } \\
\text { Currently dated around } 489 \mathrm{Ma} \text {. }\end{array}$ & $\begin{array}{l}\text { Within Bed } 23 \text { at the } 101.8 \mathrm{~m} \\
\text { level, Green Point, western } \\
\text { Newfoundland, Canada }\end{array}$ & Ratified 2000 & $\begin{array}{l}\text { Episodes } 24 \text { (1), } \\
\text { p.19-28, } 2001 .\end{array}$ \\
\hline \multicolumn{3}{|l|}{ Cambrian System } & & $\begin{array}{l}\text { Potential GSSP correlation levels } \\
\text { include Cordylodus proavus, } \\
\text { Glyptagnostus reticulatus, } \\
\text { Ptychagnostus punctuosus, Acidusus } \\
\text { atavus, and Oryctocephalus indicus. }\end{array}$ & & & \begin{tabular}{|l|} 
Overview of \\
potential \\
subdivisions in \\
Episodes $23(3)$ \\
p. $188-195,2000$ \\
\end{tabular} \\
\hline $\begin{array}{l}\text { Upper ("Furongian") } \\
\text { Series }\end{array}$ & & & & & & & \\
\hline
\end{tabular}


(Continued)

\begin{tabular}{|c|c|c|c|c|c|c|c|}
\hline $\begin{array}{l}\text { EON, Era, System, } \\
\text { Series, Stage }\end{array}$ & $\begin{array}{l}\text { Age (Ma) } \\
\text { GTS2004 }\end{array}$ & $\begin{array}{c}\text { Est. } \pm \\
\text { myr }\end{array}$ & Derivation of Age & Principal correlative events & GSSP and location & Status & Publication \\
\hline upper stage(s) in Furongian & & & & $\begin{array}{l}\text { Potential GSSP levels in upper } \\
\text { Cambrian are based on trilobites } \\
\text { and condonts }\end{array}$ & & & \\
\hline $\begin{array}{l}\text { base Paibian Stage, base } \\
\text { Furongian Series }\end{array}$ & 501.0 & 2.0 & $\begin{array}{l}\text { Radiometric ages near } \\
\text { primary marker level. } \\
\text { Estimated age and } \\
\text { uncertainty only. }\end{array}$ & $\begin{array}{l}\text { Trilobite, lowest occurrence of } \\
\text { agnostoid Glyptagnostus reticulatus. } \\
\text { Coincides with base of large } \\
\text { positive carbon-isotope excursion. }\end{array}$ & $\begin{array}{l}369.06 \mathrm{~m} \text { above base of } \\
\text { Huaqiao Fm, Paibi section, } \\
\text { NW Hunan province, south } \\
\text { China }\end{array}$ & Ratified 2003 & $\begin{array}{l}\text { Episodes article in } \\
\text { preparation }\end{array}$ \\
\hline Middle & 513.0 & 2.0 & $\begin{array}{l}\text { Radiometric ages near } \\
\text { primary marker level. } \\
\text { Estimated age and } \\
\text { uncertainty only. }\end{array}$ & $\begin{array}{l}\text { Potential GSSP levels in Middle } \\
\text { Cambrian are based mainly on } \\
\text { trilobites }\end{array}$ & & & \\
\hline Lower & & & & $\begin{array}{l}\text { Potential GSSP levels in Lower } \\
\text { Cambrian are based on } \\
\text { archaeocyatha, small shelly fossils, } \\
\text { and to a lesser extent, trilobites }\end{array}$ & & & \\
\hline \multicolumn{3}{|l|}{ PROTEROZOIC } & & $\begin{array}{l}\text { Pre-Cambrian eras and systems } \\
\text { below Ediacaran are defined by } \\
\text { absolute ages, rather than } \\
\text { stratigraphic points. }\end{array}$ & & & \\
\hline \multicolumn{8}{|l|}{ Neoproterozoic Era } \\
\hline base Ediacaran System & 630 & & $\begin{array}{l}\text { Age as suggested by } \\
\text { Ediacaran Subcomm.; } \\
\text { bracketed by } \\
\text { radiometric ages of } 600 \\
\text { and } 635 \mathrm{Ma}\end{array}$ & $\begin{array}{l}\text { Termination of Marinoan (or } \\
\text { Varanger) glaciation, and distinctive } \\
\text { C-13 change. }\end{array}$ & $\begin{array}{l}\text { Base of the Marinoan cap } \\
\text { carbonate (Nuccaleena } \\
\text { Formation), immediately } \\
\text { above the Elatina diamictite } \\
\text { in the Enorama Creek } \\
\text { section, Flinders Ranges, } \\
\text { South Australia. }\end{array}$ & $\begin{array}{l}\text { "Neoproterozoic III" } \\
\text { (ratified } 1990 \text { with } \\
\text { base defined } \\
\text { chronometrically } \\
\text { at } 650 \mathrm{Ma} \text { ) was } \\
\text { formally replaced } \\
\text { by Ediacaran } \\
\text { Period and its } \\
\text { GSSP in Feb } 2004\end{array}$ & \\
\hline Tonian System & 1000 & & $\begin{array}{l}\text { Defined } \\
\text { chronometrically }\end{array}$ & Base $=1000 \mathrm{Ma}$ & & Ratified 1990 & $\begin{array}{l}\text { Episodes } 14(2), \\
\text { p.139-140, } 1991\end{array}$ \\
\hline \multicolumn{8}{|l|}{ Mesoproterozoic Era } \\
\hline Stenian System & 1200 & & $\begin{array}{l}\text { Defined } \\
\text { chronometrically }\end{array}$ & Base $=1200 \mathrm{Ma}$ & & Ratified 1990 & $\begin{array}{l}\text { Episodes } 14(2) \\
\text { p.139-140, } 1991\end{array}$ \\
\hline Ectasian System & 1400 & & $\begin{array}{l}\text { Defined } \\
\text { chronometrically }\end{array}$ & Base $=1400 \mathrm{Ma}$ & & Ratified 1990 & $\begin{array}{l}\text { Episodes } 14(2) \\
\text { p.139-140, } 1991\end{array}$ \\
\hline Calymmian System & 1600 & & $\begin{array}{l}\text { Defined } \\
\text { chronometrically }\end{array}$ & Base $=1600 \mathrm{Ma}$ & & Ratified 1990 & $\begin{array}{l}\text { Episodes } 14(2) \\
\text { p.139-140, } 1991\end{array}$ \\
\hline \multicolumn{8}{|l|}{ Paleoproterozoic Era } \\
\hline Statherian System & 1800 & & $\begin{array}{l}\text { Defined } \\
\text { chronometrically }\end{array}$ & Base $=1800 \mathrm{Ma}$ & & Ratified 1990 & $\begin{array}{l}\text { Episodes } 14(2) \\
\text { p.139-140, } 1991\end{array}$ \\
\hline Orosirian System & 2050 & & $\begin{array}{l}\text { Defined } \\
\text { chronometrically }\end{array}$ & Base $=2050 \mathrm{Ma}$ & & Ratified 1990 & $\begin{array}{l}\text { Episodes } 14(2) \\
\text { p.139-140, } 1991\end{array}$ \\
\hline Rhyacian System & 2300 & & $\begin{array}{l}\text { Defined } \\
\text { chronometrically }\end{array}$ & Base $=2300 \mathrm{Ma}$ & & Ratified 1990 & $\begin{array}{l}\text { Episodes } 14(2) \\
\text { p.139-140, } 1991\end{array}$ \\
\hline Siderian System & 2500 & & $\begin{array}{l}\text { Defined } \\
\text { chronometrically }\end{array}$ & Base $=2500 \mathrm{Ma}$ & & Ratified 1990 & $\begin{array}{l}\text { Episodes } 14(2) \\
\text { p.139-140, } 1991\end{array}$ \\
\hline \multicolumn{8}{|l|}{ ARCHEAN } \\
\hline Neoarchean Era & 2800 & & $\begin{array}{l}\text { Defined } \\
\text { chronometrically }\end{array}$ & Base $=2800 \mathrm{Ma}$ & & Ratified 1990 & $\begin{array}{l}\text { Episodes } 14(2) \\
\text { p.139-140, } 1991\end{array}$ \\
\hline Mesoarchean Era & 3200 & & $\begin{array}{l}\text { Defined } \\
\text { chronometrically }\end{array}$ & Base $=3200 \mathrm{Ma}$ & & Ratified 1990 & $\begin{array}{l}\text { Episodes } 14(2) \\
\text { p.139-140, } 1991\end{array}$ \\
\hline Paleoarchean Era & 3600 & & $\begin{array}{l}\text { Defined } \\
\text { chronometrically }\end{array}$ & Base $=3600 \mathrm{Ma}$ & & Ratified 1990 & $\begin{array}{l}\text { Episodes } 14(2), \\
\text { p.139-140, } 1991\end{array}$ \\
\hline Eoarchean Era & & & Base is not defined & & & & \\
\hline
\end{tabular}


(2) Detailed direct ages for Upper Cretaceous ammonite zones of the Western Interior of the USA were obtained by a cubic spline fit of the zonal events and $25{ }^{40} \mathrm{Ar}-{ }^{39} \mathrm{Ar}$ dates. The base-Turonian age is directly bracketed by this ${ }^{40} \mathrm{Ar}-{ }^{39} \mathrm{Ar}$ set, and ages of other stage boundaries and stratigraphic events are estimated using calibrations to this primary scale.

(3) Seafloor spreading interpolations were done on a composite marine magnetic lineation pattern for the Late Jurassic through Early Cretaceous in the Western Pacific and for the late Cretaceous through early Neogene in the South Atlantic Oceans. Ages of biostratigraphic events were assigned according to their calibration to these magnetic polarity time scales.

(4) Astronomical tuning of cyclic sediments was used for Neogene and Upper Triassic, and for portions of the Lower and Middle Jurassic, Lower Cretaceous, and Paleocene. The Neogene astronomical scale is directly tied to the Present; the older astronomical scale provides absolute-duration constraints on polarity chrons, biostratigraphic zones and entire stages.

(5) Proportional scaling relative to component biozones or subzones. In intervals where none of the above information under Items 1 through 4 was available, it was necessary to return to the methodology employed by previous time scales. This procedure was necessary in portions of the Middle Triassic, and Middle Jurassic. Devonian stages were scaled from approximate equal duration of a set of high-resolution subzones of ammonoids and conodonts, fitted to an array of high-precision dates.

The geomathematics employed for data sets (Items 1, 2, 3 and 5) constructed for the Ordovician-Silurian, Devonian, Carboniferous-Permian, Late Cretaceous, and Paleogene intervals involved cubic spline curve fitting to relate the observed ages to their stratigraphic position. During this process, the ages were weighted according to their variances based on the lengths of their error bars. A chi-square test was used for identifying and reducing the weights of relatively few outliers with error bars that are much narrower than could be expected on the basis of most ages in the data set.

Stratigraphic uncertainty was incorporated in the weights assigned to the observed ages during the spline-curve fitting. In the final stage of analysis, Ripley's algorithm for Maximum Likelihood fitting of a Functional Relationship (MLFR) was used for error estimation, resulting in 2-sigma (95\% confidence) error bars for the computed chronostratigraphic boundary ages and stage durations. The uncertainties on older stage boundaries generally increase owing to potential systematic errors in the different radiometric methods, rather than to the analytical precision of the laboratory measurements. In this connection, we mention that biostratigraphic error is fossil event and fossil zone dependent, rather than dependent on linear age.

In Mesozoic intervals that were scaled using the seafloor spreading model or proportionally scaled using paleontological subzones, the assigned uncertainties are conservative estimates based on variability observed when applying different assumptions (see discussions in the Triassic, Jurassic and Cretaceous chapters of GTS2004). Ages and durations of Neogene stages derived from orbital tuning are considered to be accurate within a precession cycle $(\sim 20 \mathrm{kyr})$, assuming that all cycles are correctly identified, and that the theoretical astronomical-tuning for progressively older deposits is precise.

\section{Precambrian}

From the time of initial accretion and differentiation (ca. $4560 \mathrm{Ma}$ ) to the first appearance of abundant hard-bodied fossils (the onset of the Cambrian Period at $542 \mathrm{Ma}$ ), the Precambrian spans 88 percent of Earth history. Yet, there is no coherent view of a geological time scale to help describe, analyze, calibrate, and communicate the evolution of planet Earth.

The status quo is a geological time scale for the Precambrian that is both incomplete and flawed (e.g., Cloud, 1987; Crook, 1989;
Nisbet, 1991; Bleeker, 2003a), and is defined in terms of arbitrary, strictly chronometric, absolute age boundaries that are divorced from the only primary, objective, record of planetary evolution: the extant rock record.

At a recent conference in Canada on the geological time scale and its calibration (NUNA, 2003), co-sponsored by the International Committee on Stratigraphy (ICS), there was broad consensus on the view that this arbitrary, chronometrically defined, Precambrian time scale fails to convey the richness of the Precambrian rock record and therefore impedes scientific understanding of geological processes by diverting attention away from observable, first-order, stratigraphic boundaries and transitions.

Specific criticisms of the present Precambrian time scale are outlined in the chapter on Precambrian by Bleeker in Gradstein et al. (2004), but one key point deserves elaboration here: the uncertainty in decay constants of ${ }^{238} \mathrm{U}$ and ${ }^{235} \mathrm{U}$. These uncertainties (e.g., Ludwig, 2000) conspire in such a way that most age dates for the Precambrian (predominantly upper intercept ${ }^{207} \mathrm{~Pb} /{ }^{206} \mathrm{~Pb}$ zircon ages, particularly prior to $1 \mathrm{Ga}$ ) have a non-trivial fundamental "fuzziness" (e.g., about \pm 6.5 million years at ca. $2500 \mathrm{Ma}$ ). This fundamental uncertainty increases to \pm 10 million years at $4000 \mathrm{Ma}$. Definition of boundaries in terms of arbitrary, round, absolute ages, although superficially appealing, is therefore naïve. Absolute-age correlation of such boundaries between distant sections, on the basis of even our best geochronometer (U-Pb ages on single zircons), can be no better than $\pm 5-10$ million years (in terms of linear ages), even if all other sources of uncertainty (e.g., analytical scatter, $\mathrm{Pb}$ loss, or cryptic inheritance) are negligible. In principle, this fundamental uncertainty could be reduced by defining boundaries explicitly in terms of ${ }^{207} \mathrm{~Pb} /{ }^{206} \mathrm{~Pb}$ zircon ages or isotopic ratios, rather than linear age, but this would make any time scale even less transparent. Furthermore, it would not solve the problem of intercalibration between different chronometers.

Clearly, there can only be one conclusion: the Precambrian time scale should be (re)defined in terms of the only objective physical standard we have, the extant rock record. Boundaries should be placed at key events or transitions in the stratigraphic record, to highlight important milestones in the evolution of our planet. This would be analogous to the "golden spike" GSSP approach employed in the Phanerozoic. Various geochronometers (U-Pb; ${ }^{40} \mathrm{Ar}-{ }^{39} \mathrm{Ar}$; Re-Os, etc.), each with their own inherent but independent uncertainties, should be employed to calibrate meaningful stratigraphic boundaries in linear time. The ultimate result should be a calibrated "natural" time scale for planet Earth that reflects first-order events and transitions in its complex evolution.

To achieve this 'natural' time scale we propose that the 2004-2008 mandate of the International Subcommission on the Precambrian under ICS is a comprehensive and internally consistent, as well as practical, "natural" time scale for planet Earth. This 'natural' time scale should be complete with agreed upon "golden spikes" and type sections (i.e., GSSPs) for all Precambrian eon and era boundaries, and, where needed, for those of periods (systems).

Such an international effort would help focus significant attention on key stratigraphic boundaries and type sections, and, in turn, will stimulate multidisciplinary science into the causes for specific boundaries and transitions, the fundamental processes involved, their rates, and their calibration in absolute time.

Building on efforts by the previous Subcommittee on Precambrian Stratigraphy (e.g., Plumb, 1991), such a "naturalizing" of the Precambrian time scale could largely preserve existing nomenclature, in so far as it has gained acceptance in the literature, while formalizing other eon and era names that are in widespread use today, e.g. the Hadean. Thus, by 2008 , we would have, for the first time, a complete and natural time scale that reflects and communicates the entire, protracted, and complex evolution of planet Earth.

Figures 3 and 4 highlight the key points of this discussion. Figure 3 shows the formal current subdivision of the Precambrian, annotated with known key events in Earth's evolution. The practical Geon scale from Hofmann $(1990,1991)$ provides a quick chronometric shorthand notation. The interval highlighted "early Earth" is 
an informal designation commonly used for Earth's first giga-year from the time of accretion to $\sim 3.5 \mathrm{Ga}$. Exponentially decreasing impact intensity (curve on right) is schematic and includes the "late heavy bombardment" episode. Stars indicate Sudbury and Vredefort impact craters with diameters $>50 \mathrm{~km}$.

In the proposed "natural" Precambrian time scale, Earth history is divided into six eons, with boundaries defined by what can be con- sidered first-order "watersheds" in the evolution of our planet (Figure 4). The six eons can be briefly characterized as follows:

(1) "Accretion \& Differentiation" - planet formation, growth and differentiation up to the Moon-forming giant impact event;

(2) Hadean (Cloud, 1972) - intense bombardment and its consequences, but no preserved supracrustals;

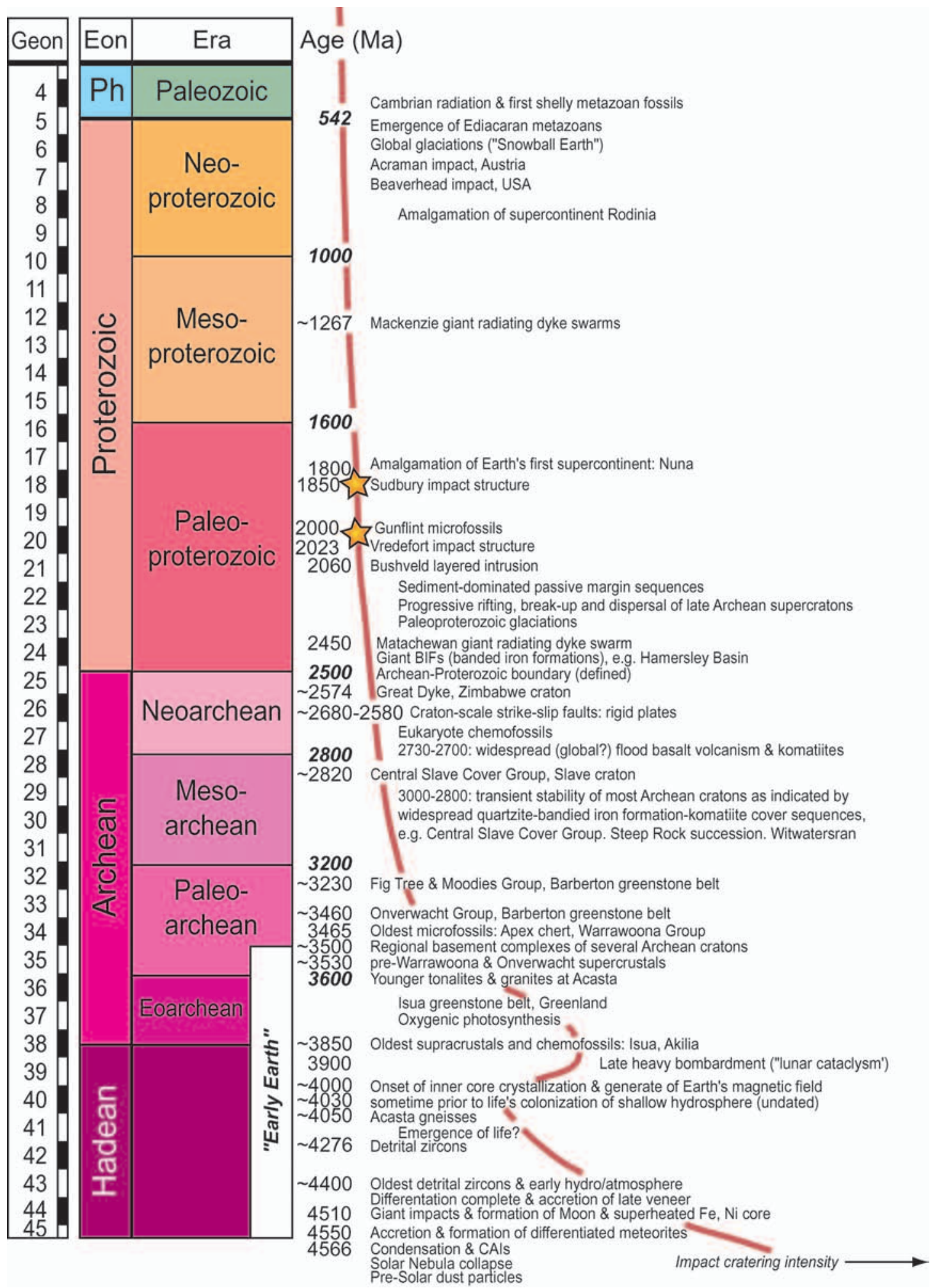

Figure 3 Formal subdivisions of the Precambrian annotated with key events in Earth's evolution. Geon scale from Hofmann (1990, 1991) provides a quick chronometric shorthand notation. 


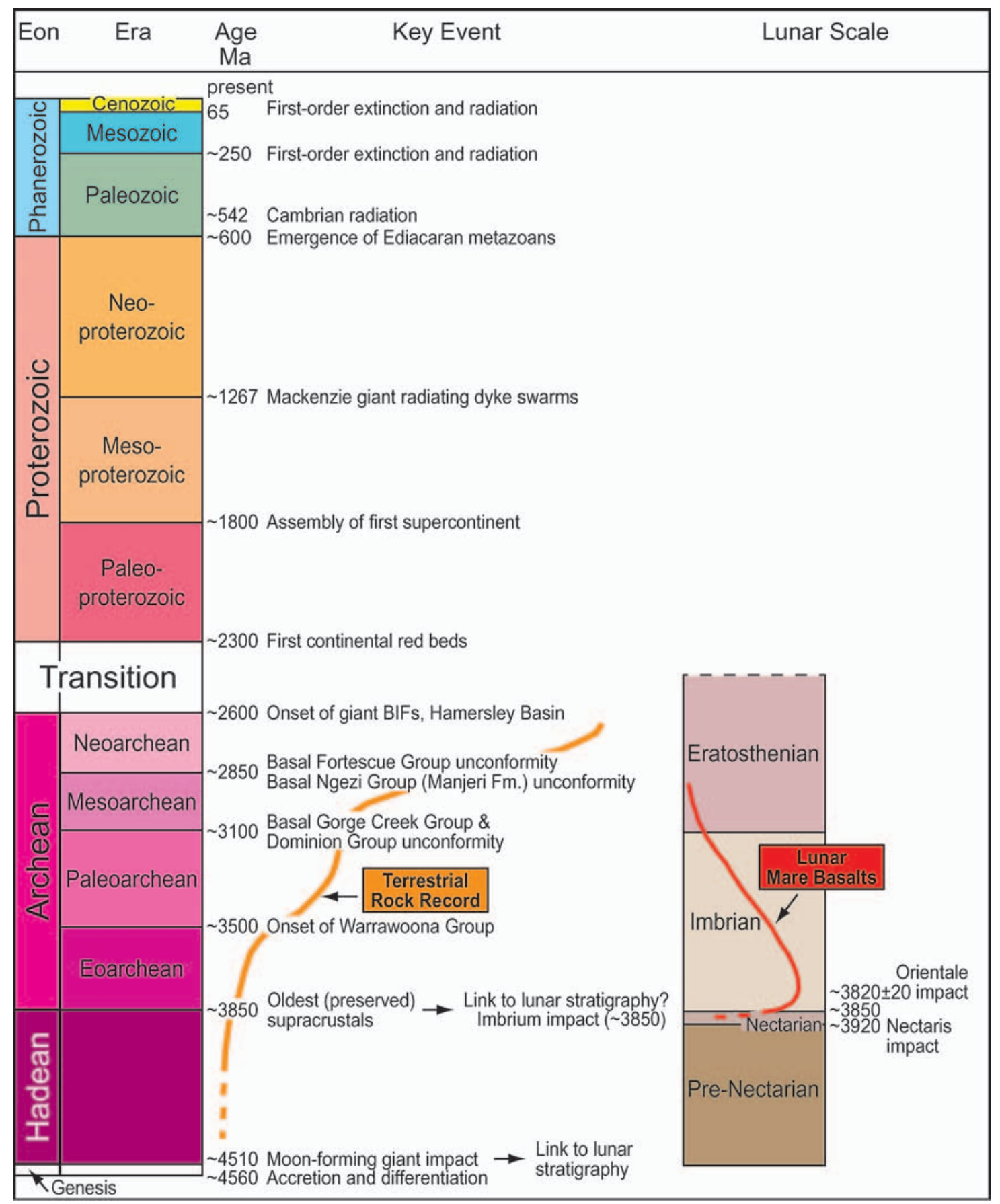

Figure 4 Proposal for a "natural" Precambrian time scale. Earth history is divided into six eons, with boundaries defined by what can be considered first-order key events in the evolution of our planet.

(3) Archean - increasing crustal record from the oldest supracrustals of Isua greenstone belt to the onset of giant iron formation deposition in the Hamersley basin, likely related to increasing oxygenation of the atmosphere;

(4) "Transition" - starting with deposition of giant iron formations up to the first bona fide continental red beds;

(5) Proterozoic - a nearly modern plate-tectonic Earth but without metazoan life, except at its very top; and

(6) The Phanerozoic - characterized by metazoan life forms of increasing complexity and diversity.

Some of the boundaries are currently poorly calibrated in absolute time, whereas the onset of the Archean should "float" with the oldest preserved supracrustal rocks, a distinction currently held by $\sim 3820-3850$ Ma rocks of the Isua greenstone belt. Comparison is shown to the lunar time scale (e.g., Guest and Greeley, 1977; Murray et al., 1981; Spudis, 1999).

\section{Neogene}

The most detailed segment of the modern geologic time scale in terms of resolution and accuracy is that for the Neogene, $23 \mathrm{Ma}$ to Recent. The subdivision of the Neogene into its constituent stages is presently well established and internationally accepted for the prePleistocene part (Table 1). New ICS task groups have been organized under the umbrella of the Subcommission on Quaternary Stratigraphy to establish an international Pleistocene subdivision of Lower, Middle and Upper, and to define the Holocene/Pleistocene 
boundary. GSSPs have been formalized for the Aquitanian (defining the Paleogene/Neogene boundary), Tortonian and Messinian stages of the Miocene, and for the Zanclean, Piacenzian and Gelasian stages of the Pliocene. In addition, the Pliocene-Pleistocene boundary has been defined.

From the 1970's until 1994, Neogene time scales were constructed using a limited number of radio-isotopic age calibration points in geomagnetic polarity sequences that were primarily derived from a seafloor anomaly profile in the south Atlantic, modified after Heirtzler et al. (1968). Biozonations and stage boundaries were subsequently tied to the resulting geomagnetic polarity time scale (GPTS), preferably via magneto-biostratigraphic calibrations
(Berggren et al., 1985). Alternatively, radio-isotopic age determinations from both sides of stage boundaries were used to calculate a best-fit radio-isotopic age estimate for these boundaries in a statistical way (chronogram method of Harland et al., 1982, 1990).

The "standard" method to construct time scales changed drastically with the advent of the astronomical dating method to the prelate Pleistocene. This method relies on the calibration, or tuning, of sedimentary cycles or cyclic variations in climate proxy records to target curves derived from astronomical solutions for the solar-planetary and Earth-Moon systems. Quasi-periodic perturbations in the shape of the Earth's orbit and the tilt of the inclination axis are caused by gravitational interactions of our planet with the Sun, the

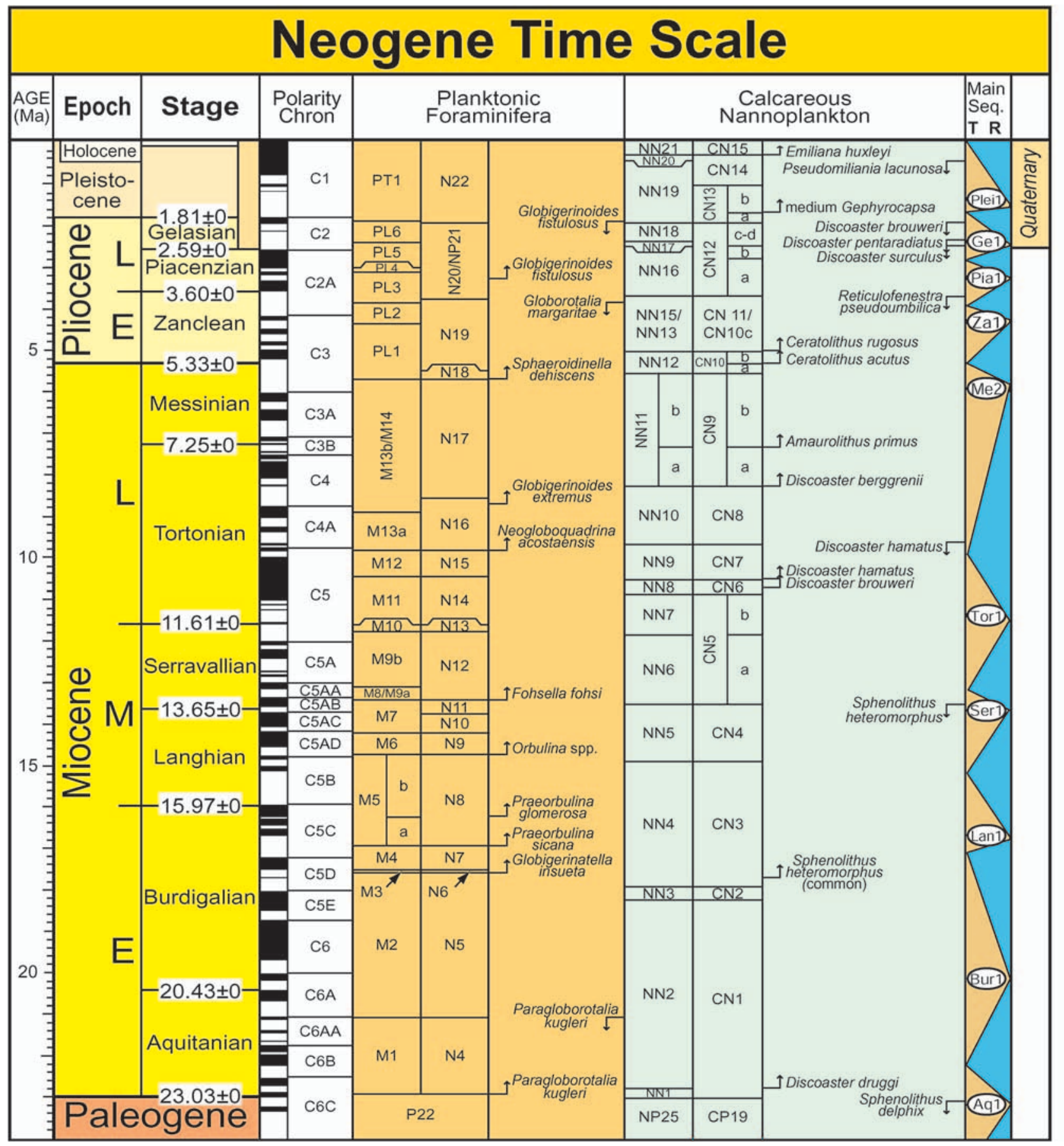

Figure 5a Neogene stratigraphic subdivisions, geomagnetic polarity scale, pelagic zonations and selected datums of planktonic foraminifers and calcareous nannoplankton. Main trends in eustatic sea level are generalized. The "Quaternary", shown schematically on the right-hand side, is traditionally considered to be the interval of oscillating climatic extremes (glacial and interglacial episodes) that was initiated at about 2.6 Ma, therefore encompassing the Holocene and Pleistocene epochs and Gelasian stage of late Pliocene. The Quaternary composite epoch is not a formal unit in the chronostratigraphic hierarchy. 
Moon and the other planets of our solar system. These interactions give rise to cyclic changes in the eccentricity of the Earth's orbit, with main periods of 100,000 and 413,000 years, and in the tilt (obliquity) and precession of the Earth's axis with main periods of 41,000 , and 21,000 years, respectively (Berger, 1977). These perturbations in the Earth's orbit and rotation axis are climatically important because they affect the global, seasonal and latitudinal distribution of the incoming solar insolation. Orbital forced climate oscillations are recorded in sedimentary archives through changes in sediment properties, fossil communities, chemical and isotopic characteristics. While Earth scientists can read these archives to reconstruct paleoclimate, astronomers have formulated models based on the mechanics of the solar-planetary system and the Earth-Moon system to compute the past variations in precession, obliquity and eccentricity of the Earth's orbit and rotation axis. As a logical next step, sed- imentary archives can be dated by matching patterns of paleoclimate variability with patterns of varying solar energy input computed from the astronomical model solutions. This astronomical tuning of the sedimentary record results in time scales based on measurable physical parameters that are independent from those underlying radio-isotopic dating and that are tied to the Recent through a direct match with astronomical curves.

Astronomical tuning was first applied in the late Pleistocene in order to build a common high-resolution time scale for the study of orbital induced glacial cyclicity. Initial attempts to extend this time scale back in time were unsuccessful due to lack of resolution or incompleteness of the sedimentary succession. These problems were overcome with the advent of the advanced piston corer (APC) technique in ocean drilling and the drilling of multiple offset holes per site. Combined these innovations were used to construct spliced

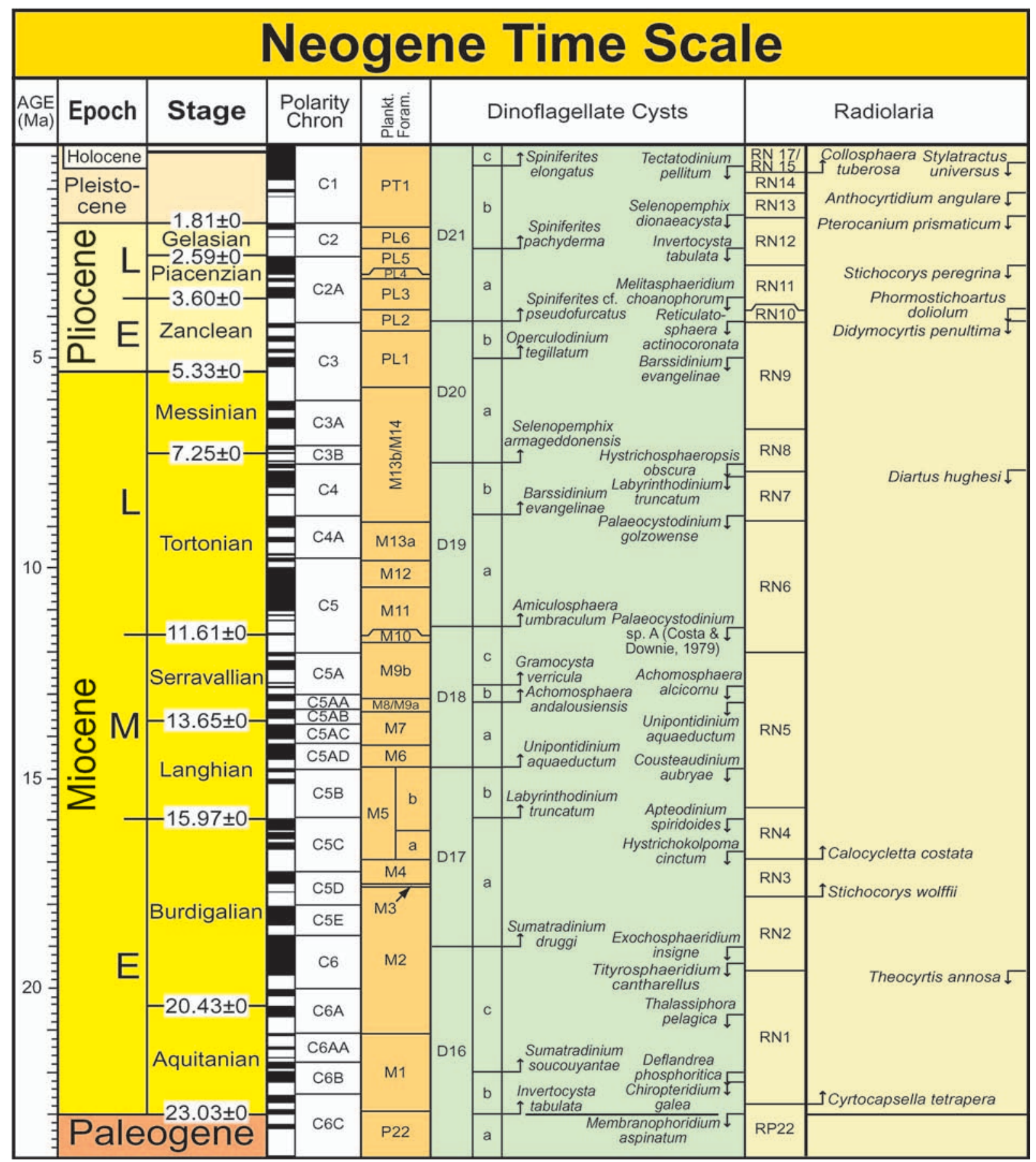

Figure $5 b$ Neogene dinoflagellate cyst and radiolarian zonation with estimated correlation to magnetostratigraphy and planktonic foraminifer zones. 
composite sections in order to recover undisturbed and complete successions marked by high sedimentation rates. Soon afterwards, the astronomical time scale was extended to the base of the Pliocene based on ODP sites (Shackleton et al., 1990) and land-based sections in the Mediterranean (Hilgen, 1991a,b), the study of the latter providing another means to overcome the problem of incompleteness of the stratigraphic record.

GTS2004 for the first time presents an Astronomically Tuned Neogene Time Scale (ATNTS2004), based on cyclic sedimentary successions from the western Equatorial Atlantic Ocean and Mediterranean. The new time scale represents a continuation of a development that led Berggren et al. (1995a) to incorporate the Pliocene and Pleistocene astrochronology of Shackleton et al. (1990) and Hilgen (1991a, b) in their Neogene time scale.

Construction of the new high-resolution Neogene time scale was made possible through:

(1) Technological and procedural improvements in deep-sea drilling of older Neogene strata,

(2) High-resolution studies of exposed marine sections in tectonically active areas where ancient seafloor has been rapidly uplifted, and

(3) Improvements in the accuracy of theoretical astronomical solutions resulting in the La2003 numerical solution.

A seafloor anomaly profile from the Australia-Antarctic plate pair was employed to complete the polarity time scale for the interval between 13 and $23 \mathrm{Ma}$ due to the lack of magnetostratigraphic records for ODP Leg 154 sites. Biostratigraphic zonal schemes are either directly tied to the new time scale via first-order calibrations, such as the standard low-latitude calcareous plankton zonation, or can be linked to it by recalibrating them to the associated polarity time scale. Formally designated chronostratigraphic boundaries (GSSPs of Neogene stages) are also directly tied to the new time scale because they are defined in sections that have been used to build the astronomically tuned integrated stratigraphic framework that underlies the time scale. An overview of the tuned Neogene stratigraphic framework is in Figures $5 \mathrm{a}$ and $\mathrm{b}$.

The new time scale resulted in a significantly younger age of 23.03 Ma for the Oligocene-Miocene boundary than the $23.8 \mathrm{Ma}$ estimated in previous time scales; the latter age was based on radiometric age determinations that are not fully acceptable according to current standards. The intercalibration of the independent astronomical and radiogenic-isotopic dating methods is not yet solved, but new results (Kuiper, 2003) point to an astronomical-derived age of $28.24 \pm 0.01 \mathrm{Ma}$ for the Fish Canyon Tuff (FCT) sanidine and favor the introduction of a directly astronomically dated standard in $40 \mathrm{Ar}-$ 39Ar dating.

The astronomically tuned Neogene time scale with an unprecedented accuracy (1-40 kyr) and resolution $(<10 \mathrm{kyr})$, opens new perspectives for paleoclimatic and paleooceanographic studies of the entire Neogene with a temporal resolution comparable to that of Pleistocene research (i.e., Krijgsman et al., 1999; Zachos et al., 2001).

\section{GTS Quo Vadis?}

The changing philosophy in time scale building has made it more important to undertake high-resolution radiometric study of critical stratigraphic boundaries, and extend the astronomical tuning into progressively older sediments. Good examples are Bowring et al. (1989) for basal-Triassic, Amthor et al. (2003) for basal-Cambrian and Hilgen et al. (2000) for Messinian. The philosophy is that obtaining high-precision age dating at a precisely defined stratigraphic boundary avoids stratigraphic bias and its associated uncertainty in rock and in time.

In this respect, it is of vital importance that ICS not only completes the definition of all stage boundaries, but also actively considers definition of standardized subdivisions within the many long stages itself. Examples of long stages (spanning more than 10 myr) that lack international standardization of internal divisions are the Campanian, Albian, Aptian, Norian, Carnian, Sakmarian, Visean, Tournaisian, Famennian and Tremadocian stages, and parts of the Cambrian system. This consensus definition process should be completed in a timely manner. Regional and philosophical arguments between stratigraphers should be actively resolved to reach consensus conclusions which focus on the global correlation implications. Stratigraphic standardization precedes linear time calibration.

Future challenges to time scale building, detailed in Gradstein et al. (2004), may be summarized as follows:

(1) Formal definition of all Phanerozoic stage boundaries, and interior definition of long stages.

(2) Orbital tuning of polarity chrons and biostratigraphic events for the entire Cenozoic and Cretaceous (past $150 \mathrm{myr}$ ).

(3) A consensus Ar/Ar monitor age (? $28.24 \pm 0.01 \mathrm{Ma}$ from orbital tuning), and consensus values for decay constants in the K-Ar isotope family.

(4) A detailed public database of high-resolution radiometric ages that includes "best practice" procedures, full error propagation, monitor ages and conversions.

(5) Resolving of zircon controversies across Devonian/Carboniferous, Permian/Triassic, and Anisian/Ladinian boundaries, either through more sampling or re-evaluation of different laboratory techniques.

(6) Detailed age dating of several 'neglected' intervals, including Upper Jurassic-Lower Cretaceous (M-sequence spreading and 'tuned' stages), base Carboniferous (Kellwasser extinction event; glaciation), and within Albian, Aptian, Norian, Carnian, Visean, and intra Permian.

(7) More detailed composite standard zonal schemes for Upper Paleozoic and Lower Mesozoic.

(8) On-line stratigraphic databases and tools (e.g., a rapid expansion of the CHRONOS network).

The geochronological science community and ICS are focusing on these challenging issues. The next version of the Geologic Time Scale is planned for the 33rd IGC in 2008, concurrent with the planned completion of boundary-stratotype (GSSP) definitions for all international stages.

\section{References}

Allègre, C.J., Manhès, G., and Göpel, C., 1995, The age of the Earth; Geochimica et Cosmochimica Acta, 59 (8), p. 1445-1456.

Amthor, J. E., Grotzinger, J.P., Schroder, S., Bowring, S.A., Ramezani, J., Martin, M.W., and Matter, A., 2003, Extinction of Cloudina and Namacalathus at the Precambrian boundary in Oman. Geology, 31 (5), p. 431434.

Berger, A., 1977, Long term variations of the Earth's orbital elements: Celestial Mechanics, 15, p. 53-74.

Berggren, W.A., Kent, D.V., and Van Couvering, J.A., 1985, The Neogene: Part 2, Neogene geochronology and chronostratigraphy, in Snelling, N.J., ed., The Chronology of the Geological Record: Geological Society of London Memoir 10, p. 211-250.

Berggren, W.A., Hilgen, F.J., Langereis, C.G., Kent, D.V., Obradovitch, J.D., Raffi, I., Raymo, M., and Shackleton, N.J., 1995, Late Neogene (Pliocene-Pleistocene) chronology: New perspectives in high-resolution stratigraphy: Geological Society of America Bulletin, 107, p. 1272-1287.

Blake, T.S., and Groves, D.I., 1987,Continental rifting and the Archean-Proterozoic transition. Geology, 15, p. 229-232.

Bleeker, W., 2003a, Problems with the Precambrian timescale: from accretion to Paleoproterozoic plate break-up. See http://www.nunatime.ca.

Bleeker, W., 2003b, The late Archean record: a puzzle in ca. 35 pieces Lithos, 71 (2/4), p. 99-134.

Bowring, S.A., Ramezani, J., and Grotzinger, J.P., 2003, High-precision U$\mathrm{Pb}$ zircon geochronology and Cambrian-Precambrian boundary. See http://www.nunatime.ca.

Cande, S.C., and Kent, D.V., 1992, A new geomagnetic polarity time scale for the Late Cretaceous and Cenozoic. Journal of Geophysical Research, 97, p. 13917-13951.

Cande, S.C. and Kent, D.V., 1995, Revised calibration of the geomagnetic polarity timescale for the Late Cretaceous and Cenozoic. Journal of Geophysical Research, 100, p. 6093-6095. 
Cloud, P., 1972, A working model of the primitive Earth. American Journal of Science, 272, p. 537-548

Cloud, P., 1987, Trends, transitions, and events in Cryptozoic history and their calibration: apropos recommendations by the Subcommission on Precambrian Stratigraphy. Precambrian Research, 37, p. 257-264.

Cooper, R.A., 1999, The Ordovician time scale - calibration of graptolite and conodont zones: Acta Universitatis Carolinae Geologica, 43 (1/2), p. 1-4.

Crook, K.A.W., 1989, Why the Precambrian time-scale should be chronostratigraphic: a response to recommendations by the Subcommittee on Precambrian Stratigraphy. Precambrian Research, 43, p. 143-150.

Gradstein, F.M., Agterberg, F.P., Ogg, J.G., Hardenbol, J., van Veen, P., Thierry, T., and Huang, Z., 1994, A Mesozoic time scale. Journal of Geophysical Research, 99 (B12), p. 24051-24074.

Gradstein, F.M., Ogg, J.G., Smith, A.G., Agterberg, F.P., Bleeker, W., Cooper, R.A., Davydov, V., Gibbard, P., Hinnov, L.A., House, M.R. (†), Lourens, L., Luterbacher, H-P., McArthur, J., Melchin, M.J., Robb, L.J., Shergold, J., Villeneuve, M., Wardlaw, B.R., Ali, J., Brinkhuis, H., Hilgen, F.J., Hooker, J., Howarth, R.J., Knoll, A.H., Laskar, J., Monechi, S., Powell, J., Plumb, K.A., Raffi, I., Röhl, U., Sanfilippo, A., Schmitz, B., Shackleton, N.J., Shields, G.A., Strauss, H., Van Dam, J., Veizer, J., van Kolfschoten, Th., and Wilson, D., 2004 (in press), A Geologic Time Scale 2004. Cambridge University Press, 500 pp

Guest, J.E., and Greeley, R., 1977. Geology on the Moon; The Wykeham Science Series, Crane, Russak and Company, Inc., New York, 235 pp.

Harland, W.B., Cox, A.V., Llewellyn, P. G., Pickton, C.A.G., Smith, A.G., and Walters, R., 1982, A geologic time scale 1982, Cambridge University Press, $131 \mathrm{pp}$

Harland, W.B., Armstrong, R.L., Cox, A.V., Craig, L.E., Smith, A.G., and Smith, D.G., 1990, A geologic time scale 1989. Cambridge University Press, $263 \mathrm{pp}$.

Heirtzler, J.R., Dickson, G.O., Herron, E.M., Pitman, W.C., and Le Pichon, X., 1968, Marine magnetic anomalies, geomagnetic field reversals, and motions of the ocean floor and continents. Journal of Geophysical Research, 73, p. 2119-2139.

Hilgen , F.J., 1991a, Extension of the astronomically calibrated (polarity) time scale to the Miocene/Pliocene boundary. Earth and Planetary Science Letters, 107, p. 349-368.

Hilgen, F.J., 1991b, Extension of the astronomically calibrated (polarity) time scale to the Miocene-Pliocene boundary. Earth and Planetary Science Letters, 107, p. 349-368.

Hilgen, F.J., Krijgsman, W., Langereis, C.G., Lourens, L.J., Santarelli, A., and Zachariasse, W.J., 1995, Extending the astronomical (polarity) time scale into the Miocene. Earth and Planetary Science Letters, 136, p. 495510 .

Hilgen, F.J., Bissoli, L., Iaccarino, S., Krijgsman, Meijer, R., Negri, A., and Villa, 2000, Integrated stratigraphy and astrochronology of the Messinian GSSG at Oued Akrech (Atlantic Morocco). Earth and Planetary Science Letters, 182 , p. 237-251.

Holmes, A., 1947, The construction of a geological time-scale. Transactions Geological Society of Glasgow, 21, p. 117-152.

Holmes, A., 1960, A revised geological time-scale. Transactions of the Edinburgh Geological Society, 17, p. 183-216.

Krijgsman, W., Hilgen, F.J., Raffi, I., Sierro, F.J., and Wilson, D.S., 1999, Chronology, causes and progression of the Messinian salinity crisis: Nature, 400, p. 652-655.

Kuiper, K.F., 2003, Direct intercalibration of radio-isotopic and astronomical time in the Mediterranean Neogene: Geologica Ultraiectina (Mededelingen van de Faculteit Geowetenschappen, Universiteit Utrecht), 235, 223 pp.

Ludwig, K.R., 2000, Decay constant errors in U-Pb Concordia-intercept ages. Chemical Geology, 166, p. 315-318.

Lumbers, S.B., and Card, K.D., 1991, Chronometric subdivision of the Archean. Geology, 20, p. 56-57.

Murray, B., Malin, M.C., and Greeley, R., 1981. Earthlike planets; surfaces of Mercury, Venus, Earth, Moon, Mars; W.H. Freeman and Company, San Francisco, 387 pp.

Nisbet, E.G., 1991, Of clocks and rocks-The four aeons of Earth. Episodes, 14, p. $327-331$

NUNA, 2003, New Frontiers in the fourth dimension: generation, calibration and application of geological timescales; NUNA Conference, Geological Association of Canada; Mont Tremblant, Quebec, Canada, March 15-18, 2003. See http://www.nunatime.ca.

Obradovich, J.D., 1993, A Cretaceous time scale, in Caldwell, W.G.E., and Kauffman, E.G., eds., Evolution of the Western Interior Basin, Geological Association of Canada, Special Paper 39, p. 379-396.

Plumb, K.A., 1991, New Precambrian time scale. Episodes, 14, p. 139-140.
Plumb, K.A., and James, H.L., 1986, Subdivision of Precambrian time: Recommendations and suggestions by the commission on Precambrian stratigraphy. Precambrian Research, 32, p. 65-92.

Remane, J., 2000, International Stratigraphic Chart, with Explanatory Note. Sponsored by ICS, IUGS and UNESCO. (distributed at the 31st International Geological Congress, Rio de Janeiro 2000), 16 pp.

Shackleton, N.J., Berger, A., and Peltier, W.R., 1990, An alternative astronomical calibration of the lower Pleistocene timescale based on ODP site 677. Transactions of the Royal Society of Edinburgh, 81, p. 251-261.

Shackleton, N.J., Crowhurst, S.J., Weedon, G.P., and Laskar, J., 1999, Astronomical calibration of Oligocene-Miocene time. Philosophical Transactions of the Royal Society of London, A, (357), p. 1907-1929.

Spudis, P.D., 1999. The Moon. In: The New Solar System, edited by J.K Beatty, C. Collins Petersen and A. Chaikin, Cambridge University Press, Cambridge, p. 125-140.

Trendall, A.F., 1991, The "geological unit" (g.u.)-A suggested new measure of geologic time. Geology, 19, p. 195.

Windley, B.F., 1984, The Archaean-Proterozoic boundary. Tectonophysics, 105, 43-53.

Zachos, J., Pagani, M., Sloan, L., Thomas, E., and Billups, K., 2001, Trends, rhythms, and aberrations in global climate $65 \mathrm{Ma}$ to present. Science, 292 , p. 686-693

Felix M. Gradstein is chair of the International Commission on Stratigraphy. Following retirement from the Geological Survey of Canada and Saga Petroleum Norway, he joined the Natural History Museum, University of Oslo as stratigraphy/micropaleontology professor, where he is developing relational stratigraphic databases for offshore Norway. His activities have included quantitative stratigraphy (he chaired previous IGCP and IUGS programs), Ocean Drilling Program legs in the Atlantic and Indian oceans, and coordinating compilation of Mesozoic and Phanerozoic geologic time scales. He is an avid skier and offshore sailor.

Jim Ogg, a professor of stratigraphy at Purdue University in Indiana USA, has been serving as SecretaryGeneral of the International Commission on Stratigraphy of IUGS since 2000. His research concentrates on the Mesozoic and Paleogene, especially paleoceanography (including ten DSDP-ODP drilling cruises), time scales of cyclic sedimentation and magnetic polarity chrons, and integrated Earth history. Gabi Ogg, his wife and fellow stratigrapher, was responsible for most of the graphics on the ICS website and in the GTS2004 book.
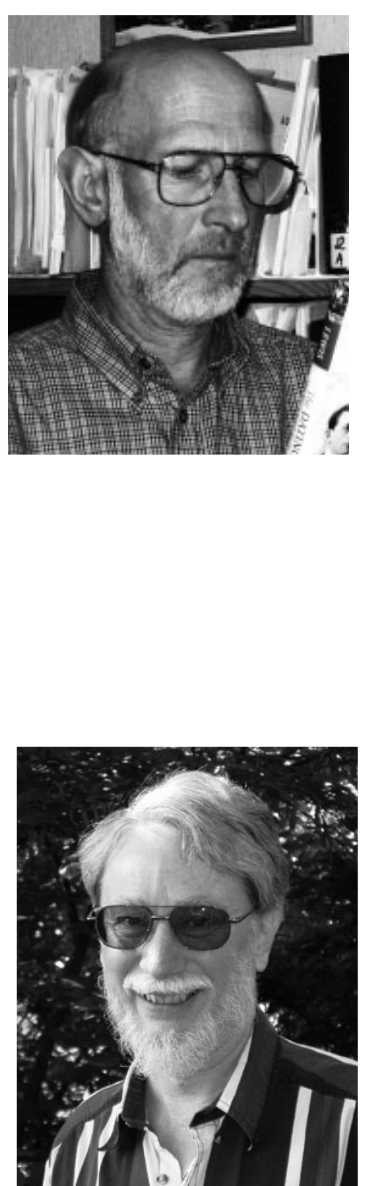